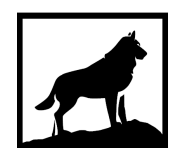

1885

Michigan

2016

\title{
A WIRELESS SENSOR SYSTEM WITH DIGITALLY CONTROLLED SIGNAL CONDITIONING CIRCUIT FOR FORCE MONITORING AT BONE FIXATION PLATES
}

Govindan Suresh

gsuresh@mtu.edu

\section{Recommended Citation}

Suresh, Govindan, "A WIRELESS SENSOR SYSTEM WITH DIGITALLY CONTROLLED SIGNAL CONDITIONING CIRCUIT FOR FORCE MONITORING AT BONE FIXATION PLATES", Open Access Master's Thesis, Michigan Technological University, 2016.

https://doi.org/10.37099/mtu.dc.etdr/247 


\title{
A WIRELESS SENSOR SYSTEM WITH DIGITALLY CONTROLLED SIGNAL CONDITIONING CIRCUIT FOR FORCE MONITORING AT BONE FIXATION PLATES
}

By

Govindan Suresh

\begin{abstract}
A THESIS
Submitted in partial fulfillment of the requirements for the degree of MASTER OF SCIENCE

In Biomedical Engineering
\end{abstract}

MICHIGAN TECHNOLOGICALUNIVERSITY

2016

(C)2016 Govindan Suresh 
This thesis has been approved in partial fulfillment of the requirements for the Degree of MASTER OF SCIENCE in Biomedical Engineering.

Department of Biomedical Engineering

$\begin{aligned} \text { Thesis Advisor: } & \text { Dr. Keat Ghee Ong } \\ \text { Committee Member: } & \text { Dr. Jingfeng Jiang } \\ \text { Committee Member: } & \text { Dr. Smitha Rao } \\ \text { Department Chair: } & \text { Dr. Sean J. Kirkpatrick }\end{aligned}$




\section{Table of Contents}

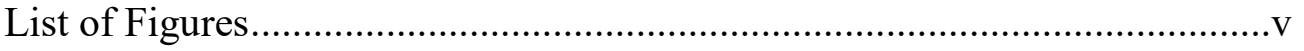

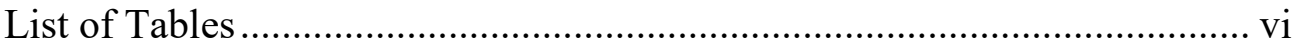

Acknowledgements ......................................................................... vii

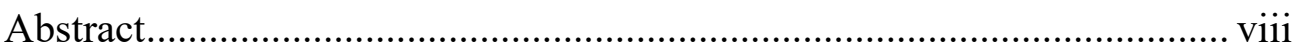

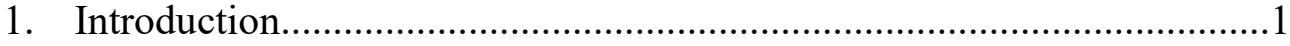

1.1. Orthopedic Trauma .................................................................

1.2. Fracture Complications............................................................

1.3. Treatment of Bone Fractures ..........................................................2

1.4. Recovery and Rehabilitation ....................................................

1.5. Limitations of Current Treatment Modality ....................................5

1.6. The Need of a Sensorized Implant .................................................6

1.7. Strain and Temperature Sensors .................................................... 9

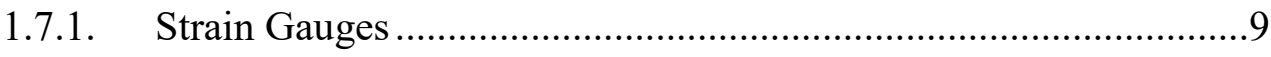

1.7.2. Criteria for Strain Gauge Selection........................................... 10

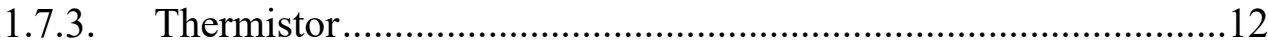

1.8. Sensor Implementation at Bone Fixation Plates............................ 12

1.9. Goals of This Project ............................................................ 14

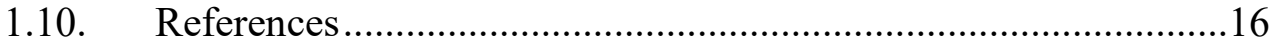

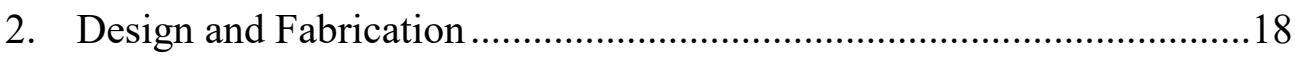

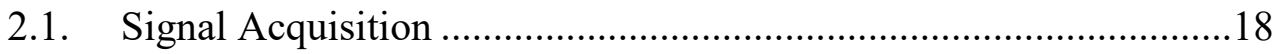

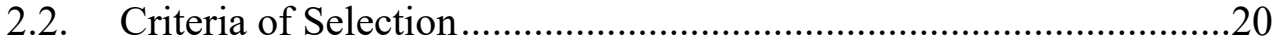

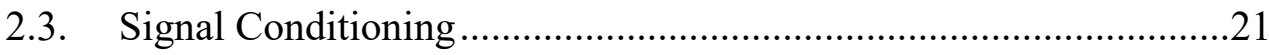

2.3.1. AD8236 Instrumentation Amplifier..........................................22

2.3.2. MCP6441 Operational Amplifier ...............................................24

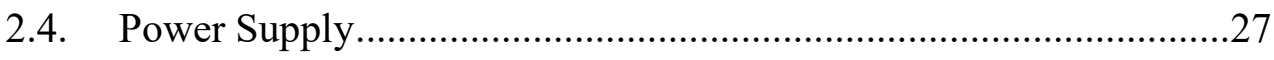

2.4.1. MCP1700T Linear Voltage Regulator......................................27

2.4.2. TLV-711 Linear Voltage Regulator..........................................29 


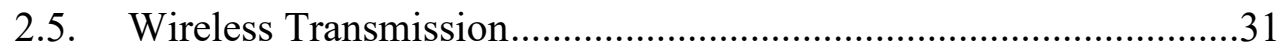

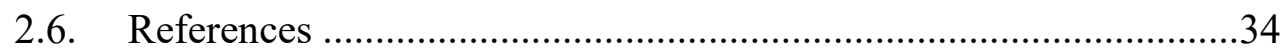

3. Digital Potentiometer to Balance Wheatstone Bridge ...........................35

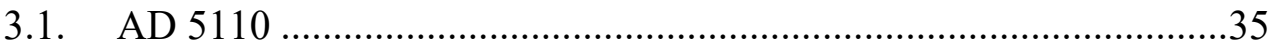

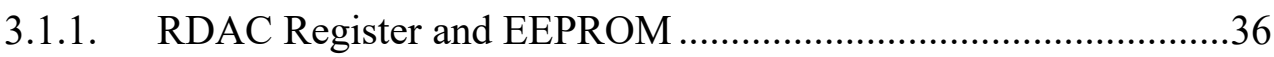

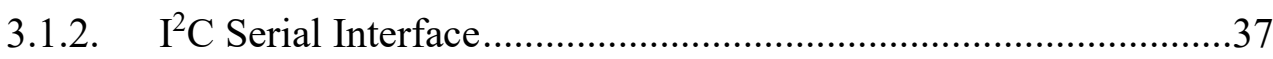

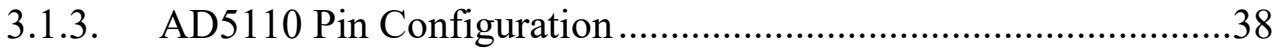

3.2. Programming the AD5110 Digital Potentiometer ...........................39

3.3. Application of AD5110 Digital Potentiometer...............................43

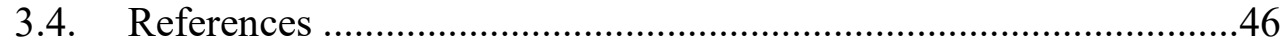

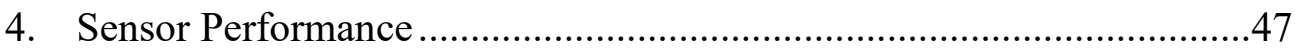

4.1 Mechanical Testing....................................................................47

4.2 Sensor Response for Compressive Loads .....................................48

4.3 Mechanical Testing Under Cyclic Loading ..................................51

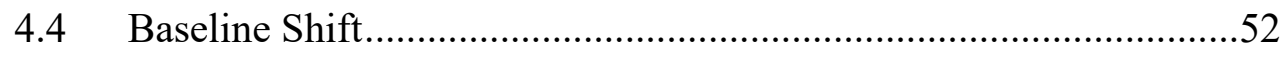

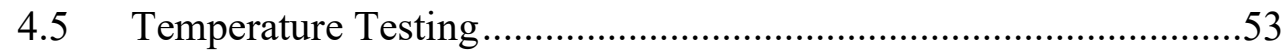

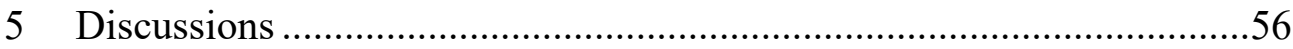

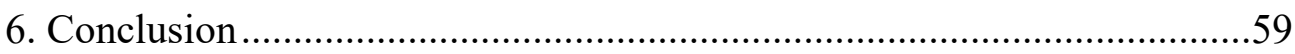

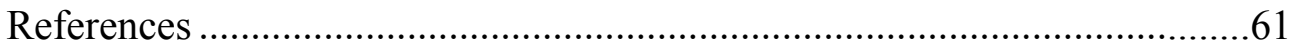




\section{List of Figures}

Figure 1.1 Schematic diagram of a bonded metallic strain gauge..................10

Figure 1.2 Image of strain gauge and thermistor glued to the outer edge of the external fixator. The fixation plate was designed and fabricated by Nicholas Quirk at Mayo Clinic

Figure 2.1 Schematic Diagram of a typical Wheatstone Bridge. $V_{E X}$ is the excitation voltage to the bridge and $R_{3}$ is the variable resistor. The output depends upon the resistance ratio of resistors in both branches 18

Figure 2.2 Schematic diagram of the Wheatstone bridge configuration used for the system 20

Figure 2.3 Schematic diagram of the Wheatstone bridge connected to an

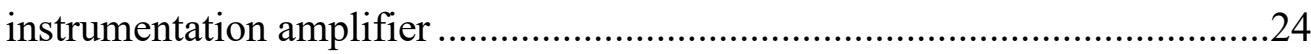

Figure 2.4 Schematic Diagram for second stage amplification ....................26

Figure 2.5 Schematic of MCP1700 voltage regulator.................................29

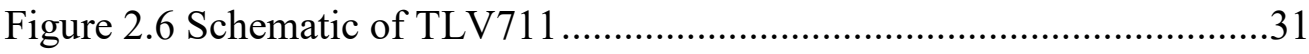

Figure 2.7 The schematic diagram of wireless signal transmission using

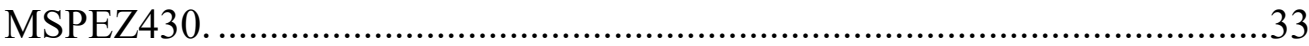

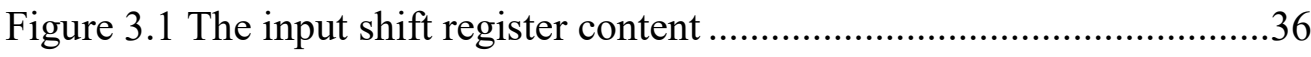

Figure 3.2 The circuit diagram of the Wheatstone bridge with AD5110 connected to a $330 \mathrm{~K} \Omega$ resistor and $324 \mathrm{~K} \Omega$ resistor (instead of two $56 \mathrm{~K} \Omega$ ). The voltage dropping across the digital pot and the upper $330 \mathrm{~K} \Omega$ resistor is fed to the non-inverting input of the instrumentation amplifier and the voltage drop across the strain gauge is applied to the inverting input. .44 


\section{List of Tables}

Table 3.1 Command Operation Truth Table ...............................................36

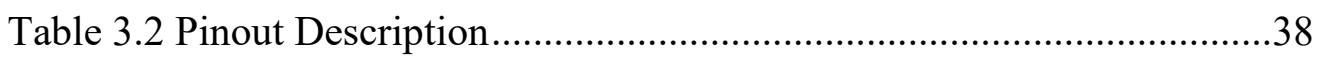

Table 3.3 Control registers of USCI module, their descriptions and user assigned values for initializing the USCI module of the MSPEZ-430 ..........40 


\section{Acknowledgements}

I would like to thank my advisor, Dr. Keat Ghee Ong for believing in me and providing me with full support and guidance throughout the research work. I would like to sincerely thank my committee members, Dr. Jingfeng Jiang and Dr. Smitha Rao, and all the faculty members in the biomedical engineering department for their teaching, help, and support. I also want to acknowledge the project group in Mayo Clinic, MN, USA for their contributions to my research work.

A special thanks to my family for their patience, support and understanding without which this was not possible. My parents and my sister, to whom I dedicate my work, have always encouraged me in my pursuit of education, dreams and happiness. I would also like to thank "Kru Nga Dow" group and all my other friends for their love and care towards me, encouragement and helping me during my stressful times. 


\section{Abstract}

Post-rehabilitation of orthopedic surgery is critical for bone fracture treatments. Current protocols are not based on quantitative assessments of the patient condition but they are conservative estimations mostly based on prior experience and physician's opinions. While there are quantitative methods for assessing the recovery of orthopedic surgery, they are typically very expensive and provide only snapshots during the healing process. A standalone, reconfigurable, embedded wireless sensor system with digitally controlled signal conditioning system capable of providing continuous monitoring of bone healing is developed. Strain sensor measurements were validated against a commercial mechanical loading instrument for relevant loads that an animal (ovine) would experience during in vivo testing (up to $250 \mathrm{~N}$ ). The loader was configured to apply a maximum force of $250 \mathrm{~N}$ to the bone fixation plate at a rate of $1000 \mathrm{~N} / \mathrm{min}$. Cyclic testing of the system showed optimal stability and no observable drift in the sensor. The sensor was also implemented in a rodent model for monitoring force loading at an internal bone fixation plate. The platform's small, robust, and low power nature is usefulness for continuous wireless monitoring and actuation in many biomedical applications. 


\section{Introduction}

\subsection{Orthopedic Trauma}

Orthopedic trauma includes a whole range of injuries from simple isolated fractures to multiple broken bones caused by lethal accidents. Many of these injuries take a long time to heal. With modern medical advancements and surgical techniques, many of these traumas are treatable to restore full functionality of the fractured section. In severe cases, especially with injuries to long bones such as the femur (thigh bone), surgical interventions along with implants are used to keep the broken bone intact as well as to provide the required mechanical support during the period of bone remodeling. The main goal of orthopedic trauma care is to allow normal healing of fractured bone while restoring the anatomical alignment of the injured section. Careful treatment including physiotherapy is required for the patient to retain full functionality [1].

Fractures account for $16 \%$ of all musculoskeletal related injuries in the US annually. More than 6.8 million people are admitted to hospitals with bone fractures. Fractures occur annually at a rate of 2.4 per 100 population [2]. Osteoporosis is a major factor of bone fracture particularly among the aging population. Specifically, adults 65 years and older constitute a higher-risk population in orthopedic trauma [2]. Due to the increase in the population and life expectancy due to the advancement in healthcare, age-related fractures such as the fragility fractures have become more common than ever [3]. The economic expenditures of orthopedic trauma are estimated to be about $\$ 230$ billion in the United States, $\$ 180$ billion in the European Union and $\$ 538$ billion worldwide. It is believed that the financial burden caused by this trauma in the respective regions are about 1 to $3 \%$ of their gross national product $[4]$.

\subsection{Fracture Complications}

Many bone fractures are accompanied by serious complications. Depending on the onset time, these complications can be classified as; early complications or late 
complications [5]. Early complications are those which arise immediately after the fracture; these may include vascular injury, visceral injury, damage to surrounding tissues, skin, nerves and wound infections. Proper and early stabilization procedures, astute nursing assessments, more directed and hasty interventions are imperative to reduce the risk related to these complications [5]. Late complications are those which occur at later stages of the treatment. Even with the most effective treatment

protocols, late complications are likely to occur depending on different factors including the site of injury, nature of the injury and the age of the patient. Majority of the late complications observed in patients with bone fractures are delayed union, mal-union, and non-union [5]. The delayed union is characterized as the failure of the fracture to consolidate within the expected time; in other words, healing process continues to take place but the outcome is uncertain [4,5]. Mal-union is a complication where the bone structure is skewed or distorted because the bone fragments join in an unsatisfactory manner compromising its functional capability [5]. Non-union is a complication wherein union has not occurred even after all the healing process have ceased, resulting in complete failure of the treatment [5]. Nonunion occurs in $1 \%$ of all the fracture complications but it tends to be more common in lower leg fractures (19\% non-union). The major factor disposing to non-union is the movement around the repair site, so during the earlier stages of the treatment, utmost importance should be given to restrict the movement near the injury site [5].

\subsection{Treatment of Bone Fractures}

Most fractures are painful, making it impossible for the patient to move the injured part. Major symptoms associated with a bone fracture include swelling and tenderness around the injury, bruising and deformity [4]. In some cases, these symptoms may be absent and further movement of the already injured site can aggravate the condition, leading to more serious fractures. Visual examinations are usually carried out by physicians to assess the overall condition of the patient as well as the extent of the injury [5]. The fracture is then evaluated using X-ray imaging 
which provides an image of the broken bone. Another imaging methodology which can be used to diagnose patients with orthopedic trauma is Magnetic Resonance Imaging (MRI). The physician can gather a much definitive assessment of the fracture condition using MRI because it is possible to get the extent of soft tissue damage, tumors and other issues relating to musculoskeletal disorders; they are most commonly used in assessing rotator cuff tears [6]. Computed Tomography (CT) is used when a high-resolution 3-dimensional image of bone pathology is needed. Based on these diagnostic methods the physician decides which protocol is needed for the treatment of the fracture [5]. In some cases, especially those of elderly and women, bone density is determined with various methods including ultrasound. All forms of treatment follow the basic rule; the fractured part is properly set and the injured area is constrained from any movement allowing the bone to heal naturally. Surgery might be necessary to avoid early complications for extremely severe cases. The common fracture treatment methods are:

1) Cast immobilization, in which a cast made up of plaster or fiberglass is used to limit movement to keep the fractured bone together, reducing chances of nonunion.

2) Functional cast or brace is generally employed where it requires limited movement of the fractured section.

3) Traction is used when proper healing requires steady, gentle pulling force while keeping the broken bone in place.

4) External fixator is used in severe cases as open fractures, which require surgery. However, surgery will produce counterproductive results if the skin and the soft tissues around the injury site are badly injured. Therefore, until a surgery is tolerable, an external fixation plate is used to hold the bone in proper position. The plates are either screwed or pinned to the bone.

5) Internal fixation, where the bone is repositioned directly after surgical procedures in its normal posture. Special internal fixation plates are used to attach the bone plates to the surface of the bone using screws or pins. Bone rods which are 
inserted down through the bone marrow are also used to hold the bone intact $[5$, $6,7]$.

\subsection{Recovery and Rehabilitation}

Numerous blood vessels (capillaries) surrounding the bone severe during a bone fracture. These severed capillaries are sealed at the site of the break by the formation of hematoma (blood clots) [5]. Within days, small capillaries start to grow into the hematoma, while the phagocytes remove dead cells and other tissue debris from the site of injury [5]. Bone reformation initiates with the proliferation of fibroblast and osteoblast and the former produces collagen fibers which seal the broken ends of the bone together, whereas the osteoblast starts to form the spongy bone. Later, in the gap between the broken bones, a repair tissue known as fibrocartilaginous callus (composed of hyaline and cartilage) will be formed which later modifies into a bony callus of spongy bone [5]. Normally, it takes almost two months for the broken ends of the bone to consolidate firmly after the fracture. Bone remodeling phase starts when the bony callus revamps itself in the presence of osteoclasts and osteoblasts. Remodeling phase generally takes many months or even years depending upon the age of the individual, nature and site of fracture [5]. Pain due to fracture ceases once the bone is reformed and the new blood vessels start supplying nutrients and other cells to the injury site [5]. The muscle strength will be diminished during the recovery stage, so it is advisable to restrict movement until the bone is solid and functional enough for normal activity [6]. Depending upon the magnitude of fracture, the bone exhibits two healing methods- primary (Intramembranous) or secondary (Endochondral healing) [7]. Intramembranous healing occurs through direct remodeling of bone across fracture by osteoclasts and osteoblasts. This occurs when fracture cortices are in direct contact with each other and associated strain is below $2 \%$ (rigid fixation) [7]. Endochondral healing occurs at sites of greater strain, through several stages of local inflammatory reactions, callus formation, and tissue remodeling. Segmental defects and open fractures heal through this secondary 
process. Pioneering studies hypothesize that reducing fixator stiffness at the right time enhanced bone healing [7]. According to this hypothesis, fracture treatment comprises of two stages: Bone healing and rehabilitation. The latter is often employed to make sure the fractured bone regains full range of motion and functionality. Physical therapy, which helps the patient to regain full functionality in a safe manner during the healing process, is an important part of fracture rehabilitation. A physical therapist works with the patient to regain fitness and to make sure that the patient can safely return to normal work and leisure activities. Returning to normal activities immediately after the recovery may increase the risk of multiple fractures so it is highly recommended to obtain the advice from a physical therapist $[6,7]$.

\subsection{Limitations of Current Treatment Modality}

The Wolff's Law states that bone in a healthy human or animal will adapt to the loads under which it is placed [8]. When the bone is subjected to a specific load, it will remodel itself over time as an adaptation mechanism (mechanotransduction), making the bone stronger to resist loading [7]. Currently, assessments are typically made by the methods discussed in the section above. These modalities provide the physicians the required assessments to plan the best course of treatment but most of them cannot be employed on a continuous basis. For example, if a bone plate is used for a patient with a fracture, it is imperative to monitor the recovery over time. However, X-ray and CT imaging are highly undesirable because these expose the patients to unwanted harmful radiations [8]. In addition, a favorable image is unable to be generated as the radiations (normal dosage) do not penetrate through the metal plate. On the other hand, MRI scans cannot be used in patients with a metallic bone plate or having electronic implants like pacemakers because the strong magnetic field generated by these machines can be fatal [9]. So, current rehabilitation protocols have proven challenging to both the physicians and the physical therapists (PTs) because it is difficult for them to get a quantitative assessment of patient's fracture condition. 
In other words, it is impossible to quantitatively monitor the fracture healing, particularly when a metallic implant is used in the treatment. Current protocols rely barely on conservative estimations based solely on the prior experience of the physicians or PTs. Numerous studies have provided overwhelming evidence that early cyclic loading of the fracture can enhance the efficacy of treatment (both range of motion and strength) [10]. The bone remodeling depends on factors like duration, magnitude and rate of loading but no studies have given a quantitative estimate of these factors. Studies are still going on to determine an accurate method to analyze bone fracture recovery. Plain radiography is commonly used to evaluate bone fusion but there are concerns on the accuracy of radiography and practicality of techniques $[9,10]$. For example, a research study conducted by Blumenthal and coworkers shows that plain radiograph assessments of bone fusion in lumbar spinal surgery have a poor agreement (accuracy $<69 \%$ ) when compared to surgical findings [11].

\subsection{The Need of a Sensorized Implant}

Post-operative rehabilitation is as critical as the surgery itself in treating orthopedic fracture. However, as discussed above, very few rehabilitation protocols rely on quantitative estimates. Instead, most of them depend on the prior experience of the surgeons, physicians, or PT's. The biggest challenge in the treatment of an orthopedic fracture is the lack of a general assessment methodology to estimate bone healing accurately. The current treatment options take into perspective only a snapshot of the healing process. A much effective treatment modality that relies on continuous bone healing with strain data can be devised using a sensorized implant. As already discussed, early loading can significantly lower recovery time and enhance bone healing but improper loading produce counterproductive results as mentioned. Hence, it is essential to determine proper loading parameters (magnitude of the load, duration of the load and rate of loading) and they vary depending on fractures and individuals. Strain at the fracture site, which is an accurate indicator of bone healing, can be interpreted from the strain on the implant. Hence, monitoring 
strain at a bone fixation plate can effectively allow us to monitor bone healing [12].

It has long been known that mechanical stimulation can enhance bone healing (Wolff's Law). Recent study evidences that controlling the surrounding mechanical environment of fractures and applying specific control can promote greater healing than previously exhibited and many of these defects will not heal without assistance from outside with fracture fixation hardware [13]. Current gold standard uses autologous grafts. However, this is not a viable treatment for all patient populations due to various co-morbidity.

Treatment options for segmental defects are scarce due to few FDA approved agents available to clinicians. One available agent that is approved is recombinant human bone morphogenetic protein-2 (rhBMP-2). This has been proven to accelerate healing of segmental defects. However, due to required supra-physiological doses, it is an unfeasible clinical option so far. Initial small rodent studies were initiated to investigate the effectiveness of rhBMP-2 use in segmental bone defects by subsequently lowering required rhBMP-2 doses [14]. During rhBMP-2 dosage investigation, modulating the mechanical environment of the defect and its healing effects were also assessed. Mechanical stimulation of the fracture gap is largely determined by fixator mechanical properties and associated interfragmentary movement (IFM) [15]. The IFM has been shown to play a huge role in initial size and quality of callus formed from initial inflammatory hematoma at fracture site [16]. Rigid fixation shows limited callus formed while loose fixation shows an increase in size. This knowledge of IFM effects has contributed to focus on the effects of dynamizing (changing/modulating) the fracture gap during the healing process, as differing levels of IFM affected different healing stages. In rodents, it was shown that lowering the dosage did not mean lesser healing outcome which was remarkable from a feasibility standpoint alone. These animal studies also showed, that dynamizing the fracture gap with controlled rhBMP-2 dose is a successful treatment of segmental defects. The authors concluded, that further optimization of timing and amount of dynamization (difference in strain across fracture defect) can be conducted 
to further improve potential benefits $[17,18]$.

Success with studies conducted on small animal models (rodents) can be carried forward to assess the efficacy in large animal models. Thus, translational studies assessing mechanical dynamization are currently in various stages of experimentation. The intrinsic differences between small and large animal studies continue to remain a stumbling block to most [19]. Large animal models are inherently expensive, complex and time-consuming when compared to small animal studies. The lengthy time-period for these studies proves to be a constraint as most rodent studies have a maximum time-period of 3 months while large animal studies (the ovine model being most common) require a minimum of 6 months (due to the healing difference between the species). During this time, the only healing information available to researchers is radiographs which on their own are difficult to obtain (small animals are much more compliant than larger brethren). Thus, the need to develop new data collection tools to assess bone healing process is inevitable. One potential method of data collection is through monitoring the physical deformation of bone fixation hardware used to fix the bone defect. Currently, several researchers are developing these protocols; all focused on internal plate fixation devices [19]. Professor Christopher Evans and his team at the Mayo Clinic, MN, USA required monitoring of their external fixator during large animal experiments. Through surface strain monitoring, it was hypothesized that fracture strain and IFM could be determined in real time. This would potentially aid in determining the healing period of the defect and optimal time to dynamize the defect.

Strain is the amount of deformation of a material when an external force is acting on it. Mathematically, strain is the ratio of change in length to the actual length, which can either be positive (during elongation) or negative (upon compression). Generally, there are four different types of strain; axial strain, bending strain, shear strain, and torsional strain [20]. When an external fixator is used to stabilize fracture, the total strain acting on the fixator will be the sum of all four-strains stated above. However, it has been found that during the initial days after implantation only the 
axial and bending strain are significant [21]. During the bone remodeling process strain on the fixator will vary and quantitatively assessing this strain variation helps to monitor bone remodeling. Thus, the primary aim of this study is to monitor the strain acting on the external fixator. Physicians and physical therapists can use the strain information as a quantitative measure of bone remodeling to make informed decisions and accurately apply an evidence-based patient care.

\subsection{Strain and Temperature Sensors}

\subsubsection{Strain Gauges}

Strain gauges are commonly used to monitor induced strain on various structures and components. A strain gauge is a device whose electrical resistance varies in proportion to the amount of strain in the device. The most commonly used strain gauges are the bonded metallic strain gauges which consists of an insulating flexible backing that supports a metallic foil arranged in a grid pattern [22]. The strain gauge is attached directly to the test specimen such that the strain experienced by the test specimen is transferred directly to the strain gauge. The strain gauge responds to the strain with a linear change in electrical resistance.

Electrical resistance of a conductor depends upon the conductor geometry. The relation between the conductor geometry and its resistance is given by the equation;

$$
R=\rho *(l / a) \quad \text { Equation (1) }
$$

Where, $\quad R$ is the resistance of the conductor,

$l$ is the length of the conductor, $a$ is the area of cross-section of the conductor and $\rho$ is the resistivity of the conductor.

The metallic strain gauge consists of a long, thin wire arranged in a grid pattern. The grid pattern maximizes the contact area such that a relatively smaller amount of stress in the direction of orientation of the foil will result in a 
multiplicatively larger change in resistance as compared to a single strand of long conductor wire.

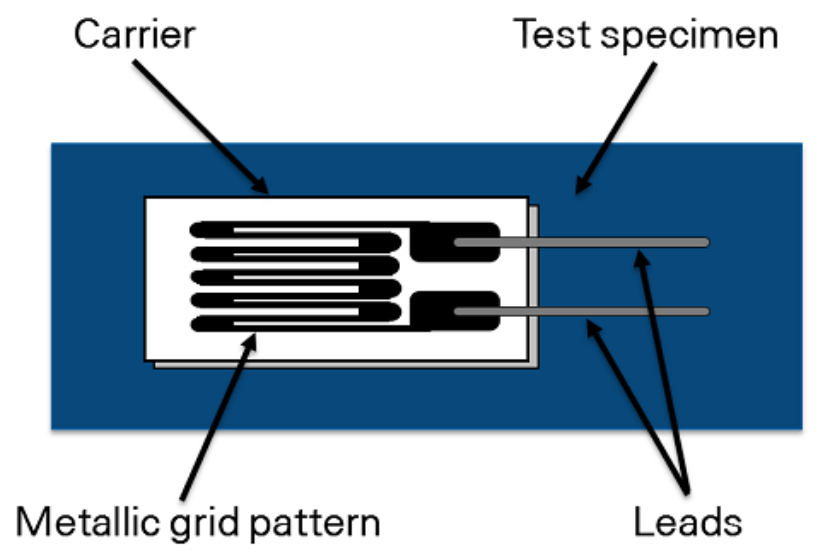

Figure 1.1 Schematic diagram of a bonded metallic strain gauge

Figure adapted from Ref. [22]

When the conductor is stretched within the limits of its elasticity, it will become narrower and longer, increasing the end to end resistance of the conductor as described in the equation (1). Similarly, the resistance will decrease if the conductor is subjected to a compressive force. Hence, the induced stress can be inferred from the measured electrical resistance of the strain gauge.

\subsubsection{Criteria for Strain Gauge Selection}

While selecting a strain gauge to monitor the stress acting on the bone implants, several parameters need to be considered, including:

- Flexibility: The system discussed here is intended to use in animal models (porcine or ovine models) and later they need to be incorporated for humans. A surgically induced long-bone defect will be stabilized by an external fixator plate equipped with the strain sensor system. The movement of the injured section induces strain on the fixator plate and the strain gauge must be flexible enough to track the strain caused by different animal movement (motion 
while walking, running, sitting and so on).

- Mechanical Strength: The most important function of the external fixator plate is fracture stabilization by providing a mechanical support for the bone during the remodeling phase. The strain gauge should have adequate mechanical strength so that they can withstand an applied load without failure.

- Temperature Stability: The resistance of a conductor varies with temperature. As the strain gauge is exposed to surrounding temperature, any change in temperature will affect the strain gauge response. The strain gauge that can give stable output irrespective of any change in temperature must be selected for the specific application.

- Sensitivity: The fundamental parameter of the strain gauge is its sensitivity to strain, expressed quantitatively as the gauge factor (GF). Gauge factor is defined as the ratio of fractional change in electrical resistance to fractional change in length.

- Adhesion to substrate: As already discussed, the strain gauge need to be adhered to the surface of the bone fixation plate. The strain gauge should be easily affixed with cold or hot curing adhesives. Also, the strain gauge must withstand usual sterilization processes.

Omega Precision SGD-3/350-LY13 are linear, miniaturized strain gauges commonly used for experimental stress analysis application. Their rugged construction and flexibility make them suitable for accurate static and dynamic strain measurement. The grid dimensions are $13 \mathrm{~mm} \times 7.20 \mathrm{~mm}$ surrounded by $22 \mathrm{~mm} \times$ $10 \mathrm{~mm}$ carrier. The measuring grid is formed by etching Constantan foil, that is completely sealed in a polyamide based carrier medium. The nominal resistance of the strain gauge is $350 \Omega$ with a gauge factor of 2.1 (tolerance level of $\pm 5 \%$ ). They are highly stable and known to produce stable reliable outputs at a broad temperature range [23]. 


\subsubsection{Thermistor}

The thermistor is a type of resistor whose resistance is dependent on temperature. They are commonly used as temperature sensors, in self-regulating heating elements, over-current protectors, and current limiting circuits. Thermistors can be subdivided into two types; thermistors with negative temperature coefficient (NTC-resistance decreases with increase in temperature) and those with positive temperature coefficient (PTC-resistance increases with the decrease in temperature). They are typically made up of ceramics or polymers and they achieve a greater precision than resistance temperature detectors within a limited temperature range $\left(-90^{\circ} \mathrm{C}\right.$ to 130 $\left.{ }^{\circ} \mathrm{C}\right)[24]$. A PTC thermistor with a nominal resistance of $47 \Omega$ is attached next to the strain gauge to monitor the temperature of the bone fixator plate. The strain gauge response varies with temperature which can be measured by the thermistor. The temperature data can be used to calibrate the strain gauge response.

\subsection{Sensor Implementation at Bone Fixation Plates}

A wireless sensor allows the clinician to observe a longer and detailed course of the injury, allowing them to make recommendations such as what physical activities the patient should avoid and whether the mechanical integrity of the implant is failing. A strain gauge based sensor system capable of continuously tracking the strain acting on an external bone fixator plate is designed and tested. The sensor system consisted of a wireless communication circuit, a signal conditional circuit (to convert resistance variations of sensors to voltages), a power supply (1/2-AA battery), a strain gauge, and a thermistor (for an external fixation plate). For the external fixation plate, the strain gauge and thermistor were attached to the bone fixation plate. The external fixation plate $(1.7 \mathrm{~cm}(\mathrm{~L}) \times 0.20 \mathrm{~cm}(\mathrm{~W}) \times 0.20 \mathrm{~cm}(\mathrm{H})$ was composed of two identical metal posts that fit securely within each other. This plate was designed to be applied to a sheep. Six metal pins traverse through the fixation plate to secure the device to the bone fracture. Appropriate positioning of the strain gauge on the bone fixation plate is essential to maximize the sensitivity. 
On average, the center of the bone fixation plate would receive the maximum strain when a load is applied. So, the strain gauge was placed in the center of the fixator plate at the side that was facing away from the bone (see Figure 1.2). In addition, the strain gauge was adhered closer to the edge of the fixator surface (as shown in Figure 1.2 at the right side of the fixation plate) because tests on the left side (replacing the thermistor) showed a 10x decrease in signal strength. Before attaching the components, the surface of the external fixator is cleaned with Kim wipe lint-free tissue paper soaked in isopropyl alcohol to remove all forms of debris over the plate surface. Once the surface was cleaned, a thin layer of adhesive (Loctite 401) was applied to the surface of the fixation plate. The strain gauge and the thermistor were then adhered to the surface and left to dry at room temperature for 3 hours before the test. To reduce the risk of sensor detachment, electrode breakage, physical damage, and exposure to liquids, heat, and moisture, multiple layers of plastic dip (Performix Plasti Dip) was applied over the adhered components.

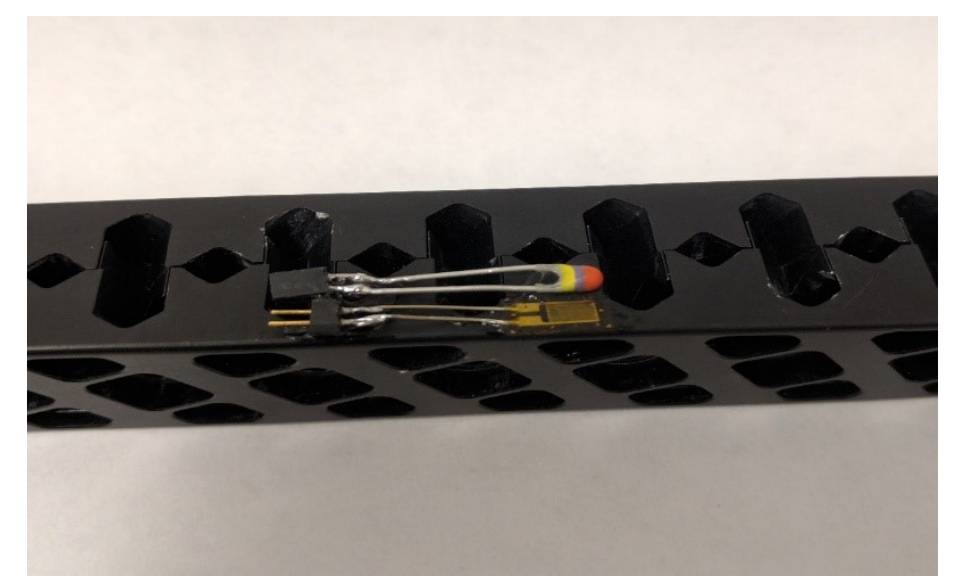

Figure 1.2 Image of strain gauge and thermistor glued to the outer edge of the external fixator. The fixation plate was designed and fabricated by Nicholas Quirk at Mayo Clinic.

The internal plate as shown in Figure 1.3 was applied to a rat model. An Omega strain gauge with a nominal resistance of $353 \Omega$ was embedded inside the structure of the plate $(24.5 \mathrm{~mm} \times 4.7 \mathrm{~mm} \times 3.3 \mathrm{~mm})$ and two leads from the strain gauge 
protrudes outside the plate which can be connected to the sensor system. This fixation plate is designed for stabilizing a critical size defect $(>6 \mathrm{~mm})$ fracture of a femur segmental defect of a rat. With the strain gauge embedded, the force loading at the fixation plate will be able to be monitored post-implantation.

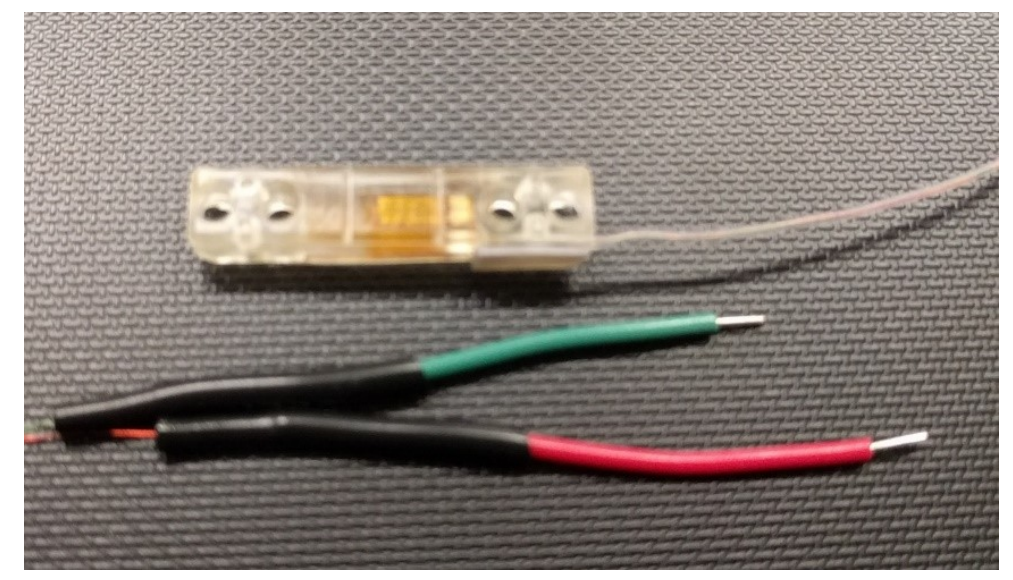

Figure 1.3. Internal plate for a femur segmental defect for rat. The plate was designed and fabricated by Brett Klosterhoff at Georgia Tech.

For conducting experiments the external fixator was used. The signal conditional circuit and the wireless communication circuit were embedded inside an enclosure and the battery was placed inside another separate enclosure to minimize the interference with the metal fixator posts. The strain gauge was selected as a transducer due to its small physical dimension and ease to integrate with the fixator plate surface. Strain acting on the fixator plate changes the resistance of the strain gauge and a custom designed electronic circuitry with a Wheatstone bridge converts this change in resistance into corresponding voltage. The signal conditioning section of the circuit filters and amplifies the voltage signal and it is wirelessly transmitted to a PC using a microcontroller. The values are calibrated and displayed using a customizable Matlab GUI.

\subsection{Goals of This Project}


The focus of this project is to design a customized strain gauge based wireless sensor system to quantitatively monitor strain due to tensile and compressive forces applied on an external or internal bone fixation plate.

The main objectives of this project are listed below:

1) Further improve the sensor system for monitoring the strain acting on a fixator plate (internal and external).

2) Incorporate a digitally controlled signal conditioning circuit by using a digital potentiometer to balance the input of the Wheatstone bridge circuit at zero strain.

3) Evaluate the performance of the sensor system in terms of sensitivity, stability, noise, and temperature dependency. 


\subsection{References}

1. Gundle KR "Reticulations of orthopedic Surgery: The Process of Specialty Boundary Formation and the Provision of Fracture Care in the United States". Lulu: 2014. ISBN 1312540451

2. Riddle, Daniel L., and Susan M. Schappert. "Volume of ambulatory care visits and patterns of care for patients diagnosed with plantar fasciitis: a national study of medical doctors". Foot \& ankle international 25.5 (2004): 303-310.

3. Dawson-Hughes, Bess, et al. "Effect of calcium and vitamin D supplementation on bone density in men and women 65 years of age or older". New England Journal of Medicine 337.10 (1997): 670-676.

4. Skinner, H.B "Treatment in Orthopedics". Current Diagnosis \&amp; 2014, McGraw-Hill

5. "The-musculoskeletal-system-38/bone-216/bone-remodeling-and-repair-81912062 ", Boundless-biology-textbook.

6. http://skagitnorthwestortho.com/education/physical-therapy/bone-fractures/

7. Huang, Chenyu, and Rei Ogawa. "Mechanotransduction in bone repair and regeneration". The FASEB Journal 24.10 (2010): 3625-3632.

8. Wolff, Julius. "The law of bone remodeling”. Springer Science \& Business Media, 2012.

9. Skinner, Harry B. "Current diagnosis \& treatment in orthopedics". Lange Medical Books, McGraw-Hill Medical Publishing Div., 2006.

10. J Luke, MA. Shanahan, H. Lebowitz, and B. Holton "Current Medical Diagnosis \&amp; Treatment 2016”. Editors. 2015, McGraw-Hill Education.

11. Wachs, Rebecca A., et al. "Elementary Implantable Force Sensor: For Smart Orthopaedic Implants". Advances in biosensors and bioelectronics 2.4 (2013).

12. Blumenthal, Scott L., and Kevin Gill. "Can Lumbar Spine Radiographs Accurately Determine Fusion in Postoperative Patients?: Correlation of Routine Radiographs with a Second Surgical Look at Lumbar Fusions". Spine 18.9 (1993): 1186-1189. 
13. Claes, L. E., and J. L. Cunningham. "Monitoring the mechanical properties of healing bone". Clinical Orthopaedics and Related Research ${ }^{\circledR} 467.8$ (2009): 1964-1971.

14. Glatt, Vaida, et al. "Improved healing of large segmental defects in the rat femur by reverse dynamization in the presence of bone morphogenetic protein-2". $J$ Bone Joint Surg Am 94.22 (2012): 2063-2073.

15. Epari, D. R., et al. "A case for optimising fracture healing through inverse dynamization”. Medical hypotheses 81.2 (2013): 225-227.

16. Fountain, Stephanie, et al. "Monitoring Healing Progression and Characterizing the Mechanical Environment in Preclinical Models for Bone Tissue Engineering". Tissue Engineering Part B: Reviews 22.1 (2015): 47-57.

17. Gardner, Trevor Noel, and Sanjay Mishra. "The biomechanical environment of a bone fracture and its influence upon the morphology of healing". Medical engineering \& physics 25.6 (2003): 455-464.

18. Glatt, Vaida, et al. "Improved healing of large segmental defects in the rat femur by reverse dynamization in the presence of bone morphogenetic protein-2". $J$ Bone Joint Surg Am 94.22 (2012): 2063-2073.

19. Reichert, Johannes C., et al. "The challenge of establishing preclinical models for segmental bone defect research", Biomaterials 30.12 (2009): 2149-2163.

20. Rees, David. Basic engineering plasticity: an introduction with engineering and manufacturing applications. Butterworth-heinemann, 2012.

21. Van den Beukel, A. "Theory of the effect of dynamic strain aging on mechanical properties". Physica status solidi (a)30.1 (1975): 197-206.

22. http://www.ni.com/white-paper/3642/en/.

23. http://www.omega.com/pressure/pdf/SGD_LINEAR1-AXIS.pdf.

24. McGee, Thomas Donald. Principles and methods of temperature measurement. John Wiley \& Sons, 1988. 


\section{Design and Fabrication}

\subsection{Signal Acquisition}

A Wheatstone bridge is an electrical circuit used to measure an unknown electrical resistance by balancing two legs of a bridge circuit, one leg of which includes the unknown component. The main advantage of the Wheatstone bridge is its ability to provide extremely accurate measurements as compared to a voltage divider circuit [1]. Strain measurements rarely involve quantities larger than few milli-strains so the change in resistance of the strain gauge is measured in Wheatstone bridge configuration. The Wheatstone bridge is the electrical equivalent of two voltage divider circuits connected with an excitation voltage $\left(V_{E X}\right)$ as shown in

\section{Figure 2.1.}

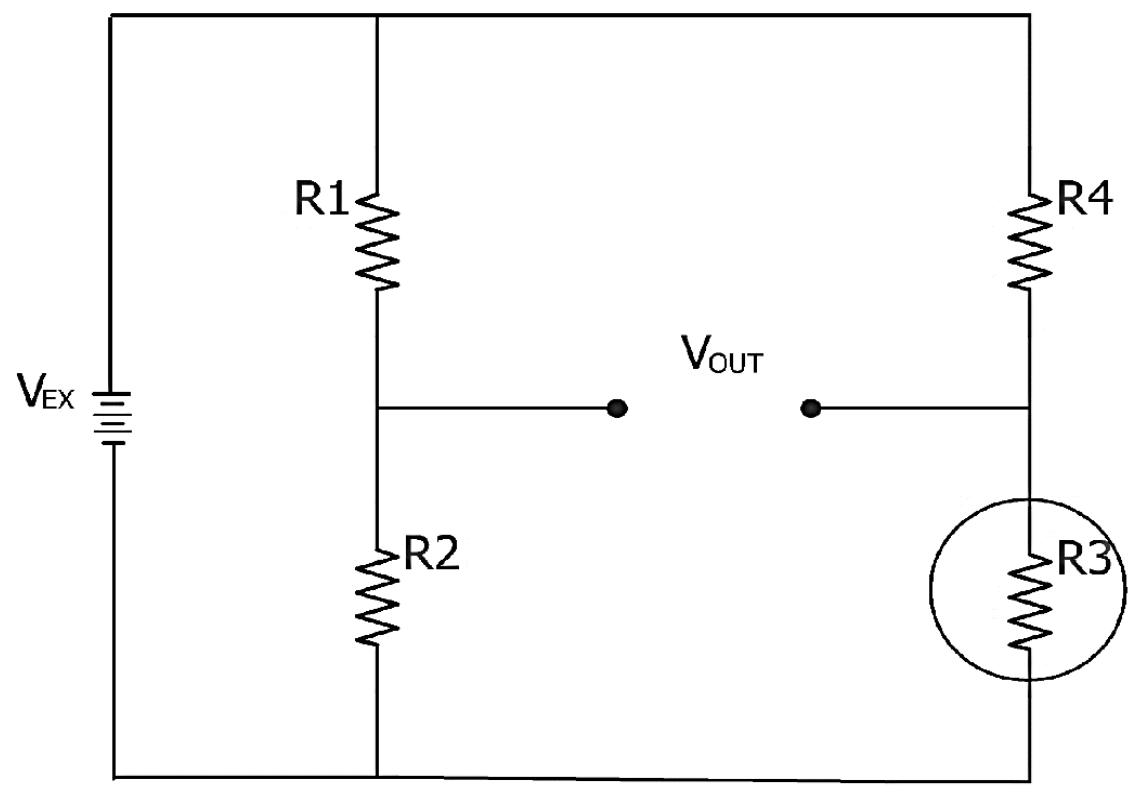

Figure 2.1 Schematic Diagram of a typical Wheatstone Bridge. $V_{E X}$ is the excitation voltage to the bridge and $R_{3}$ is the variable resistor. The output depends upon the resistance ratio of resistors in both branches

$R_{1}$ and $R_{2}$ are the resistors in one voltage divider circuit and $R_{3}$ and $R_{4}$ compose 
the second voltage divider circuit. The output of the Wheatstone bridge, VOUT is measured across the middle nodes of the two voltage dividers.

$$
V_{\text {OUT }}=\left[\frac{R_{3}}{R_{3}+R_{4}}-\frac{R_{2}}{R_{1}+R_{2}}\right] * V_{E X} \quad \text { Equation (1) }
$$

From the equation given above, the bridge is balanced if the ratio of resistance $R_{I}$ and $R_{2}$ equals to that of the ratio of $R_{3}$ and $R_{4}$. Any change in resistance in any of the four resistors will give a positive output voltage (VOUT). If one of the resistors is replaced by a strain gauge, then any change in the strain gauge resistance will unbalance the bridge to produce a non-zero output voltage which will be a function of the strain [1].

A Wheatstone bridge is designed to acquire the strain gauge signal. A $330 \mathrm{~K} \Omega$, $324 \mathrm{~K} \Omega$ and a $10 \mathrm{~K} \Omega$ digital potentiometer were connected in voltage divider configuration as shown in Figure 2.2. The strain gauge with the nominal resistance of $350 \Omega$ and a $340 \Omega$ resistor act as the second voltage divider circuit and the excitation voltage for the bridge circuit is $1.25 \mathrm{~V}(1 \mathrm{~V} 25)$. The voltage dropping across the strain gauge (V1) and the cumulative drop (V2) across the potentiometer and the $330 \mathrm{~K} \Omega(\mathrm{RA} 5)$ are fed to the inputs of an instrumentation amplifier. 


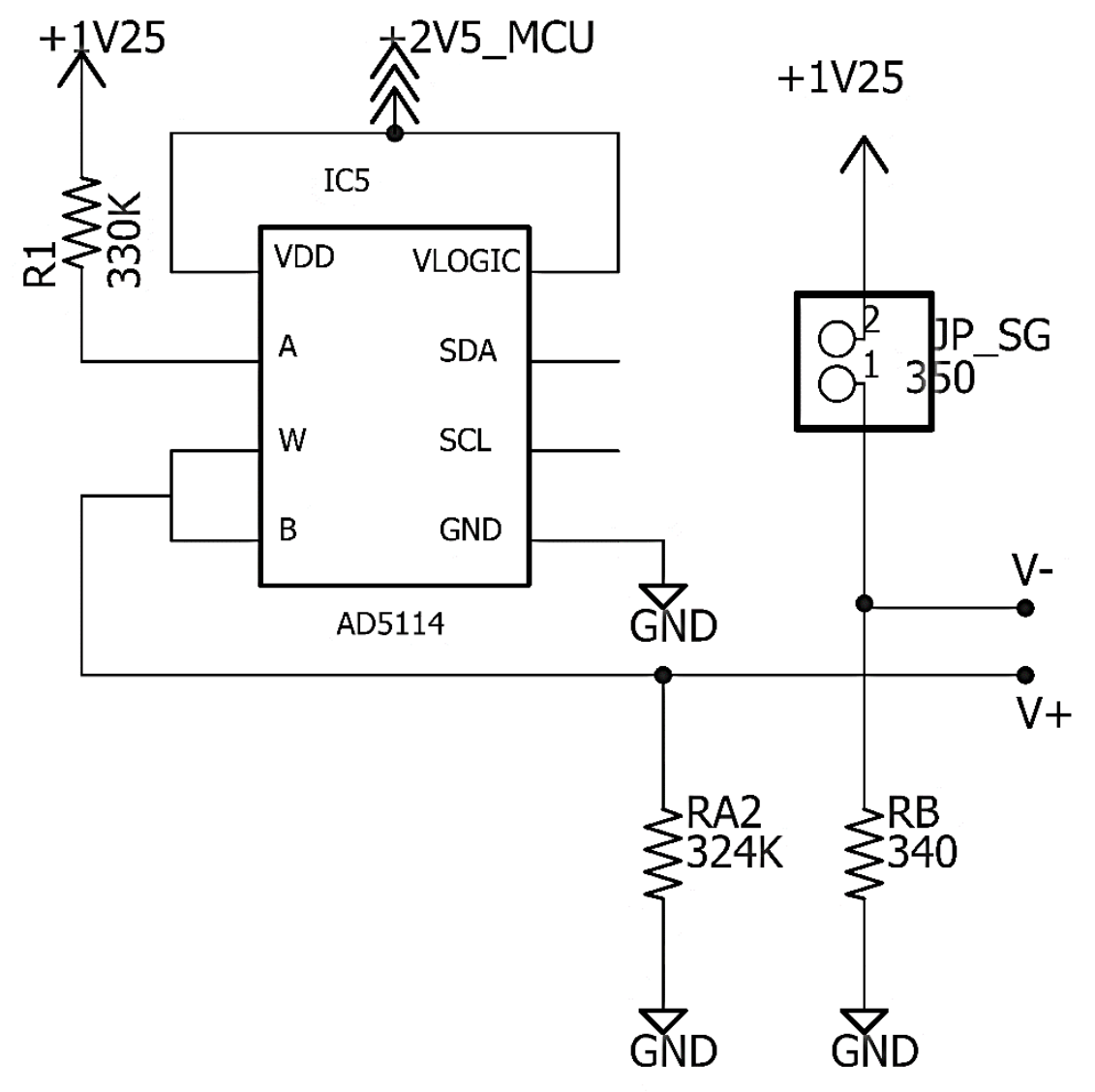

Figure 2.2 Schematic diagram of the Wheatstone bridge configuration used for the system

The $10 \mathrm{~K} \Omega$ potentiometer connected in series to the $330 \mathrm{~K} \Omega$ resistor is used to balance the bridge. The potentiometer reduces the error caused by the resistor tolerance and it also enables us to set an output offset voltage. Any voltage above the set voltage level corresponds to the change in resistance of the strain gauge.

\subsection{Criteria of Selection}

To balance the bridge circuit, the ratio of resistances in both the voltage divider circuits should be equal. The strain gauge is placed in the upper branch of one of the 
voltage divider circuits whose lower branch comprises a $340 \Omega$ resistor. The ratio of resistances of this voltage divider circuit is found to be 1.029. Thus, the second voltage divider circuit should have a comparable resistance ratio to ensure accurate measurement. A $330 \mathrm{~K} \Omega$ resistor is connected in series with a $10 \mathrm{~K} \Omega$ digital potentiometer in the upper branch and a $324 \mathrm{~K} \Omega$ resistor in the lower arm comprises the second voltage divider circuit of the Wheatstone bridge. Both the lower arm resistors are connected to the ground as shown in Figure 2.2. Mathematically, the ratio of resistance varies from 1.018 (potentiometer value is zero) to 1.049 (potentiometer full scale). The resistance ratio of the first voltage divider circuit (1.029) lies within the range provided by the potentiometer. Another important factor to be considered while selecting the components is the temperature coefficient, which is defined as the resistance change factor per unit change in temperature (Celsius). The components with low temperature coefficient is preferred for this application, so that their intrinsic property won't change with temperature variations. Hence we chose components with temperature coefficient $\pm 25 \mathrm{ppm} /{ }^{\circ} \mathrm{C}$ for our application.

\subsection{Signal Conditioning}

The strain gauge signal is transmitted wirelessly to a PC using a microcontroller. However, the strain gauge output has very low voltage levels which make it impossible for the microcontroller to process the strain data [2]. So, proper signal conditioning must be carried out to replicate the original signal. Signal conditioning includes filtering, amplification, isolation, and all other processes require to make the strain gauge voltage suitable for signal processing.

Filtering and amplification are the two important signal conditioning functions. Amplification brings up the voltage level to a range required for the microcontroller to process. It also enhances the resolution and the signal to noise ratio of the system. Not all the signal frequency spectrum contains useful data and these unwanted components (noise) will distort the original signal when amplified. (For example, power line interference at $50 / 60 \mathrm{~Hz}$ will produce noise if amplified). Hence, the noise 
needs to be filtered out of original signal before amplification [3].

\subsubsection{AD8236 Instrumentation Amplifier}

An instrumentation amplifier is commonly used in most of the precision medical devices. An optimum instrumentation amplifier must have high common mode rejection ratio, high input impedance, low output impedance, high slew rate and low power requirement. The instrumentation amplifier does not require any external feedback resistors for their operation instead they have laser trimmed resistors fabricated onto the IC itself. The gain of the amplifier can be customized to the desired value using a single gain setting resistor [4]. The strain gauge used in the system is floating (no direct connection to the ground) and floating components often create noise in the signal [5]. The instrumentation amplifier can faithfully amplify a floating signal because it only amplifies the difference between the two input terminals. They offer high-level noise rejection and minimal loading effect due to their high input impedance.

AD8236 is a monolithic, two op-amp instrumentation amplifier suitable for low power portable applications. The inclusion of rail-to-rail input and output stage offers

a wide dynamic range of operation and the novel architecture of the op-amp internally boosts the supply rail, allowing the amplifier to work rail to rail with very small non-linearity (5ppm). The supply voltage requirement of these amplifiers is as low as $1.8 \mathrm{~V}$ and it only draws a maximum of $40 \mu \mathrm{A}$ current for its operation, thus making it an excellent choice for battery-powered applications. Precise, laser timed resistors provide a high common mode rejection ratio $(C M R R=110 \mathrm{~dB})$. resistors

The output of the AD8236 depends on the potential at the reference terminal (pin 6) and in the difference in voltage between the inverting (-IN) and non-inverting $(+\mathrm{IN})$ inputs. The reference pin can be connected to a voltage that is referenced to the local ground allowing us to offset the output of AD8236 to a voltage level other than the local ground. This makes the amplifier compatible to interface with an ADC which typically cannot handle voltages close to the ground. 
The output voltage equation for the instrumentation amplifier is given by

$$
V o=G *(V n i n v-V i n v)+\text { Vref } \quad \text { Equation (3) }
$$

Where, $\quad G=$ gain

Vninv $=$ voltage across the non-inverting terminal

Vinv $=$ voltage across the inverting terminal.

Vref $=$ Voltage in the reference pin.

The gain can be selected by placing an appropriate resistor across the RG terminals (pin $2 \& 3$ ). The gain resistor can be calculated using the equation:

$$
R_{G}=420 K \Omega /(G-5) \quad \text { Equation (4) }
$$

The default gain of the amplifier is 5 when no gain resistor is connected to the terminals. The accuracy of the gain depends on the tolerance of the gain resistor $\left(R_{G}\right)$ so it is recommended to use a resistor with a very low tolerance range. The AD 8236 has very high power supply rejection ratio (PSRR) to reduce the effects of noise. However, it is recommended to use a stable dc output to power up the instrumentation amplifier for optimal performance. All the terminals of the AD8236 are protected from Electrostatic discharge (ESD) internally. The AD 8236 also have $3.1 \mathrm{pF}$ gate capacitor to eliminate low-frequency RF interference but to eliminate high-frequency interferences low pass RC network need to be placed at the inputs of the instrumentation amplifier $[4,6]$. 


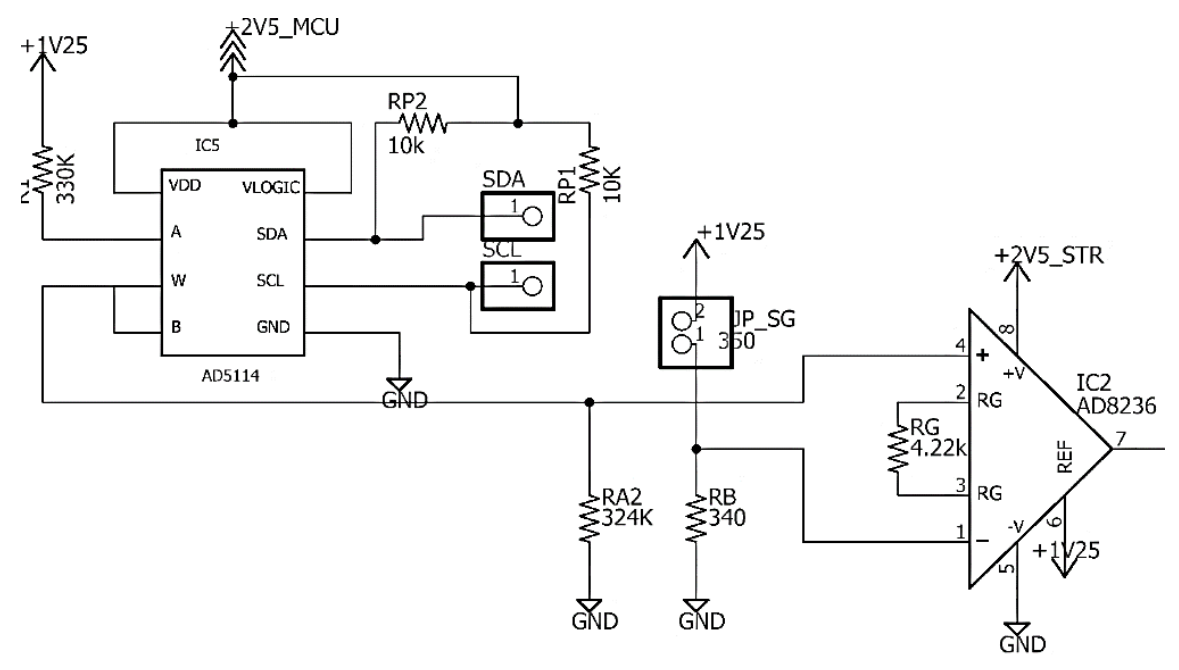

Figure 2.3 Schematic diagram of the Wheatstone bridge connected to an instrumentation amplifier

A steady voltage of $2.5 \mathrm{~V}$ (2V5_STR) from the voltage regulator power up the amplifier and a voltage of $1.25 \mathrm{~V}$ (1V25) is applied to the reference terminal (pin 6). The gain of the instrumentation amplifier is selected as 100 (AI) by connecting a $4.22 \mathrm{~K} \Omega$ resistance across the $\mathrm{RG}$ (pin $2 \& 3$ ) terminals. The output of the strain gauge (JP_SG 350) is connected to the inverting input (pin 1) of the instrumentation amplifier and the voltage dropping across potentiometer $\left(R_{3}, 2 K \Omega\right)$ and the upper $56 \mathrm{~K} \Omega$ resistor of the Wheatstone bridge is applied to the non-inverting terminal (pin 4). The output (pin 7) of the amplifier is fed to an operational amplifier for the second stage of amplification.

\subsubsection{MCP6441 Operational Amplifier}

The gain provided by the instrumentation amplifier alone is not sufficient for the proper processing of the signal. Due to the low output impedance of the instrumentation amplifier huge current will be drawn if the output from the instrumentation amplifier is fed directly to the microcontroller, because of the low impedance of the load. Thus, the second stage of amplification is required for the 
faithful reproduction of the signal. The MCP6441 is a single nano power operational amplifier with a low quiescent current (450nA) and with rail to rail input and output operations. An important feature of this amplifier is that they can operate with a single supply voltage as low as $1.4 \mathrm{~V}$. The input stage of the MCP6441 op-amp uses two differential input stages in parallel where one operates at a low common mode input voltage (VCM) and the other at a high VCM. The device operates with a VCM up to $300 \mathrm{mV}$ above VDD and $300 \mathrm{mV}$ below VSS. The output voltage range of MCP6441 is VSS +20mV (minimum) and VDD -20mV (maximum) when the load ( $\mathrm{RL} \sim 10 \mathrm{~K} \Omega$ ) is connected to the maximum allowable supply voltage. The op-amp is designed to eliminate phase reversal when the input pins exceed supply voltage. An inbuilt electrostatic discharge (ESD) protection network protects the input transistors of the op-amp against power surges and during harmful static discharges [7].

As depicted in the figure, the diodes clamp the inputs whenever the voltage drops below VSS. Static interferences usually occur at low voltages so, clamping diodes are used in conjunction with VSS to cancel the effects of interferences. However, these diodes clamp any voltages that go well beyond VDD. 


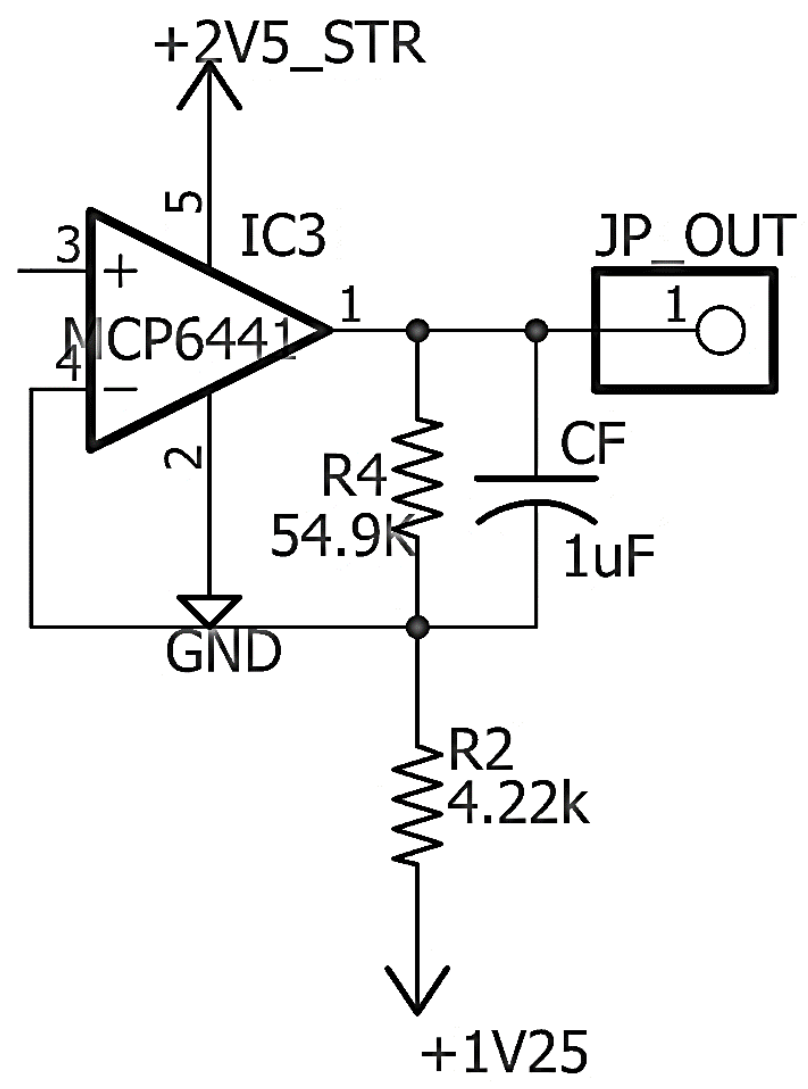

Figure 2.4 Schematic Diagram for second stage amplification

The MCP6441 is powered by supplying $2.5 \mathrm{~V}$ (2V5_STR) to its VDD terminal (pin 5) and pin 2 is connected to the ground. The op-amp is used in voltage follower configuration with a gain. The gain of a non-inverting op-amp is given by the equation:

$$
A_{V}=1+\left(R_{4} / R_{1}\right)
$$

Equation (5)

In the schematic shown above $R_{2}$ and $R_{4}$ are the gain resistors and the gain of the second amplification stage is selected as 13.42 using resistors $54.9 \mathrm{~K} \Omega\left(R_{F}\right)$ and $4.22 \mathrm{~K} \Omega\left(R_{1}\right)$. Thus, the overall gain of $1342\left(A_{I} \times A_{V}\right)$ is achieved after the signal amplification stage. A $0.1 \mu \mathrm{F}$ capacitor is connected at the output to bypass the 
leakage current to ground.

\subsection{Power Supply}

The components in the board need to have constant and stable power supply to perform the optimum function and the power requirements vary depending upon the components. The components can be seriously damaged if the power surges meanwhile, low voltages may not be able to power up a component to its full functionality [8]. A voltage regulator generates a fixed output voltage of a preset magnitude that remains constant regardless of changes to its input voltage or load conditions. Linear voltage regulators are used for providing the stable power supply for the devices in the system. A linear regulator employs an active (BJT or MOSFET) pass device (series or shunt) controlled by a high gain differential amplifier. It compares the output voltage with a precise reference voltage and adjusts the pass device to maintain a constant output voltage. For example, a 3.6V Lithium-ion battery cell (Xeno XL-050F/T2 1/2 AA 3.6V) is used to power up the board. These primary, non-rechargeable batteries are specifically designed for long term applications at a higher performance. High and steady operation voltage, improved transient minimum voltage effect and very low discharge rate make them suitable for our application. For internal plate, a coin cell (CS2032) is use. CS2032, which has a power capacity of about $240 \mathrm{mAh}$, has the best power-to-size ratio.

The strain gauge based sensor system have two important functions, it collects the strain data and it also transmits the collected data to a PC with the help of a microcontroller. Apart from providing power to the circuit component's the voltage regulators should also power up the end device microcontroller attached to the circuit board to facilitate wireless data transmission. Two separate voltage regulators were used in the system to fulfill the power requirements of the sensor system.

\subsubsection{MCP1700T Linear Voltage Regulator}

The MCP $1700 \mathrm{~T}$ is a family of CMOS low dropout (LDO) voltage regulator that 
can deliver $250 \mathrm{~mA}$ at $2.5 \mathrm{~V}$. It can regulate the output voltage even when the supply voltage is as close to the output voltage. The input operating range for this device is specified from $2.3 \mathrm{~V}$ to $6 \mathrm{~V}$ making it an excellent choice for single cell battery powered applications.

The input of the MCP1700T is connected to the source of a P-channel PMOS pass transistor. A $1 \mu \mathrm{F}$ ceramic capacitor needs to be connected between the input and ground terminal to ensure low source impedance and is necessary for regulator stability. A portion of the output from the pass transistor is fed back to an internal error amplifier and is then compared with a reference voltage. Any change in the input voltage will cause the error amplifier to respond and the output of the error amplifier adjusts the amount of current that flows through the pass transistor, thus regulating the output voltage to the desired value. In the event of a short circuit or excessive output current, the internal circuitry of the MCP1700T will turn off the Pchannel transistor for a short time and it will automatically restart once the normal condition is reached (overcurrent protection). A thermal shutdown protection circuitry (within the IC) will turn off the device if the device temperature reaches a certain threshold. The power dissipation of the regulator IC depends upon the load current and on the differential voltage of input and output. The internal junction temperature increases if the power dissipation within the pass transistor increases. The internal junction threshold is set at $140^{\circ} \mathrm{C}$ at that point, the device shut down and begin to cool down to the normal temperature levels protecting the device from catastrophic failure [9]. 


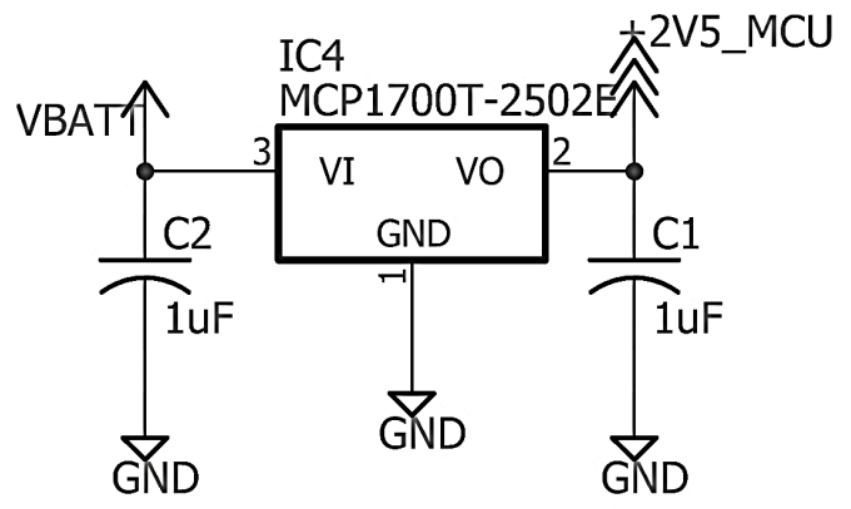

Figure 2.5 Schematic of MCP1700 voltage regulator

An unregulated input of 3.6V from a Lithium-ion battery is given to the input pin (pin 3 ) of the voltage regulator and to ensure regulator stability a $1 \mu \mathrm{F}$ ceramic capacitor is connected across the input and the ground pin. The regulated output (2V5_MCU) from the VOUT pin (pin 2) is fed to the VIN port of the microcontroller, thereby powering the end-device microcontroller. The VOUT pin is also connected to the ground through a $1 \mu \mathrm{F}$ ceramic capacitor to ensure the stability of the output.

\subsubsection{TLV-711 Linear Voltage Regulator}

The TLV-711 are series of dual, low dropout (LDO) voltage regulators with excellent line and load transient performance. They are low quiescent current $(35 \mu \mathrm{A})$ devices designed for power-sensitive applications. These devices are able to provide an accuracy of $2 \%$ over temperature. These regulators provide regulated output voltages of $2.5 \mathrm{~V}$ and $1.25 \mathrm{~V}$ if an input voltage ranging from $2 \mathrm{~V}$ to $5.5 \mathrm{~V}$ is applied to the IC. Thermal and over-current protection circuitry are also included inside the package to prevent damage to IC during normal operations. Another important feature of TLV-711 voltage regulators is the presence of an active pull-down circuit that quickly discharge the output. They also have pull-down resistors connected to 
the enable pins which help in disabling the output pins of the device. The enable pins can be used to switch OFF the device when the sensor system is not in use. By default, the voltage regulator is ON, it can be switched OFF by giving a high to low transition signal to the enable pins.

The input pin (pin 2) is connected to the source of two P-channel PMOS pass transistor. The IC package houses two voltage regulators providing two outputs at the same time. A portion of the output from both regulators are fed to two separate error amplifiers. Both the error amplifiers have dedicated VREF, the error amplifiers compare the output of the pass transistors with the reference voltages. If the input voltage varies, the error amplifiers adjust the output of the pass transistor thereby regulating the output voltage to desired levels. The presence of dedicated VREF for each output highly diminishes the chance of cross-talk interference. The current limiter circuits turn OFF the pass transistors during power surges protecting both the device and all other components connected to it. The device is enabled if a voltage above $0.9 \mathrm{~V}$ appears across the enable pins (pin $1 \& 3$ ) and it can be turned OFF by connecting the enable pin with a voltage less than $0.4 \mathrm{~V}$. An active high in the enable pins triggers the 'Enable and power control logic' circuit of the regulator IC to switch ON the pass transistor and the device deliver regulated outputs through the output pins (pin $5 \& 6$ ). Whenever the temperature at the pass transistor junction exceeds a threshold, the thermal shutdown circuit detect the rise in temperature and sends a signal to the logic circuit which turns OFF the device until the device temperature reaches normal level. An under-voltage lockout circuit (UVLO) keep the output shut off until the internal circuitry is operating properly [10]. 


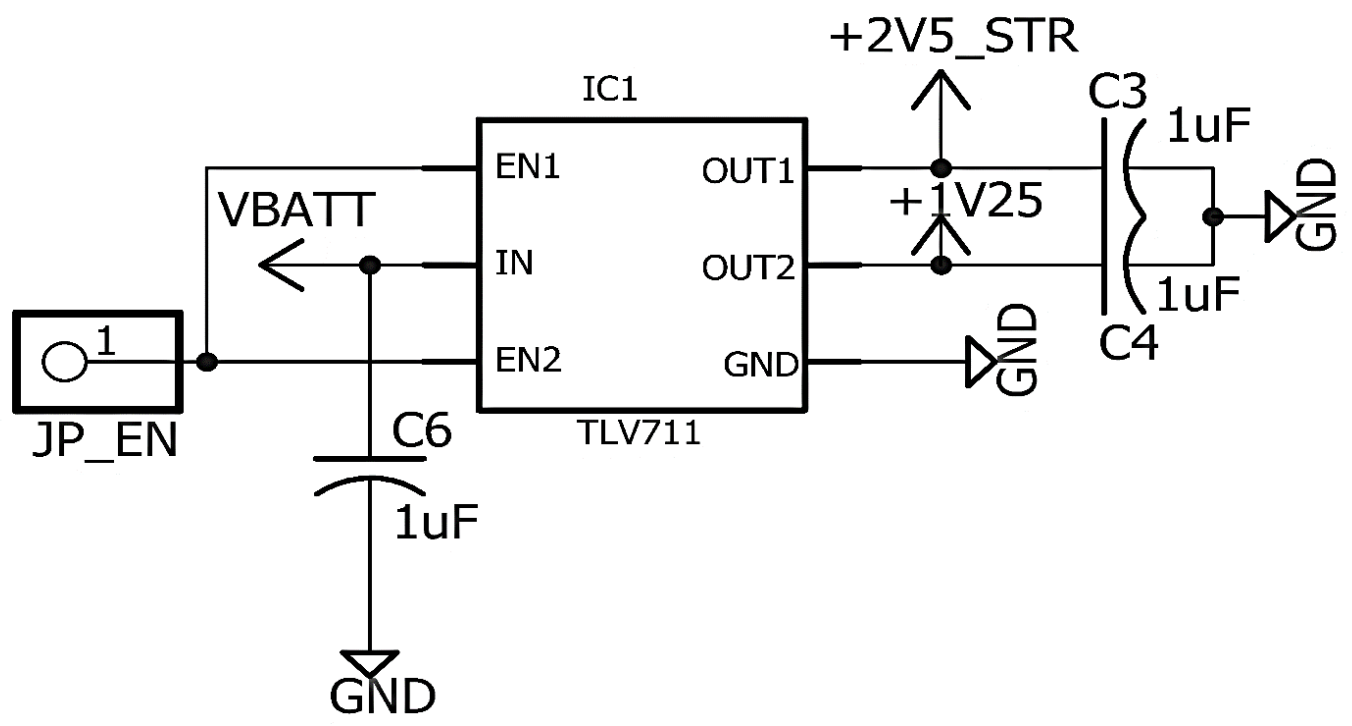

Figure 2.6 Schematic of TLV711

An unregulated voltage of $3.6 \mathrm{~V}$ (VBATT) is supplied to the input pin (IN) of the TLV-711 from the Lithium-ion battery source. The input pin is connected to the ground through a $1 \mu \mathrm{F}$ ceramic capacitor (C6). This capacitor ensures device stability as it counteracts reactive input sources and improves transient response, noise rejection and ripple response. Both the enable pins are shorted together and connected to port 1.2 of the end-device microcontroller. The regulator can be turned OFF by sending a low signal to the enable pins. The enable pin (JP_EN) is connected to the end device microcontroller which controls the state of the device. Output 1 (OUT1) power up the amplifiers (instrumentation amplifier and op-amp) in the circuit and the second output (OUT2) provides the virtual ground voltage for all the components in the circuit. Both the outputs of the regulator are connected to the ground through $1 \mu \mathrm{F}$ ceramic capacitors $(\mathrm{C} 3$ and $\mathrm{C} 4)$ to ensure output stability.

\subsection{Wireless Transmission}

Wireless communication technology has become an integral part of several types 
of communication devices as it allows users to communicate even from remote areas. Some of the advantages of wireless communication include flexibility, cost effectiveness, constant connectivity, convenience and enhanced efficiency. Various modes of wireless communication exist and some obvious factors need to be considered while selecting a specific wireless technology. This list includes:

1) Range: To estimate the distance between the transmitter and the receiver, or whether the distance is fixed or changing. If the distance varies what are the maximum and minimum distances.

2) Duplex or simplex: Is the system need to communicate in two-way (duplex) or is the application one-way (simplex).

3) Number of Nodes: Usually in simple systems, there will be only one transmitter and a receiver which can be termed as a two-node communication system. If the system includes multiple transmitters and receivers, then they are known as multimodal system. In the multi-nodel system, we need to estimate the total number of nodes and must define the mode of interaction between them.

4) Data rate: It denotes the speed at which the data transfer occurs. Usually, for monitoring and control a low data rate is required as compared to high speed for video transfer. Link reliability and noise immunity will be high for communication in low data rate.

5) Interferences: Interferences can be caused by a variety of reasons. These may include environmental factors such as physical obstacles can block or reflect a signal leading to signal loss. If there are other wireless devices nearby and the noise from machinery, power lines can all act as sources of interference.

6) Power Source: Usually for battery powered applications we need to consider the type of battery, the size, life, recharging needs, battery replacement intervals, and related costs. How the inclusion of wireless technology increases the power consumption of application also should be estimated.

7) Size and space: This was the most important criteria to be checked while 
designing our sensor system. The wireless system should take up as little as space as possible [11].

The EZ430-RF2500 is a complete wireless development tool for the MSP430 and CC2500 that includes all the hardware and software required to develop an entire wireless project with the MSP430 in a convenient USB stick. The tool includes the highly integrated MSP430F2274 ultra-low-power MCU with two 2.4-GHz wireless target boards. The eZ430-RF2500 uses the MSP430F2274 which combines 16-MIPS performance with a 200-ksps 10-bit ADC and 2 op-amps and is paired with the CC2500 multi-channel RF transceiver designed for low-power wireless applications. The tool also includes a USB powered emulator to program and debug the application in the system. Wireless communication is carried out by a simple, low power RF network protocol known as SimpliciTI [12]. They are well suited for such networks that contain battery-powered devices long battery life, low data rate and low duty cycle and have a limited number of nodes talking directly to each other or through an access point or range extenders.

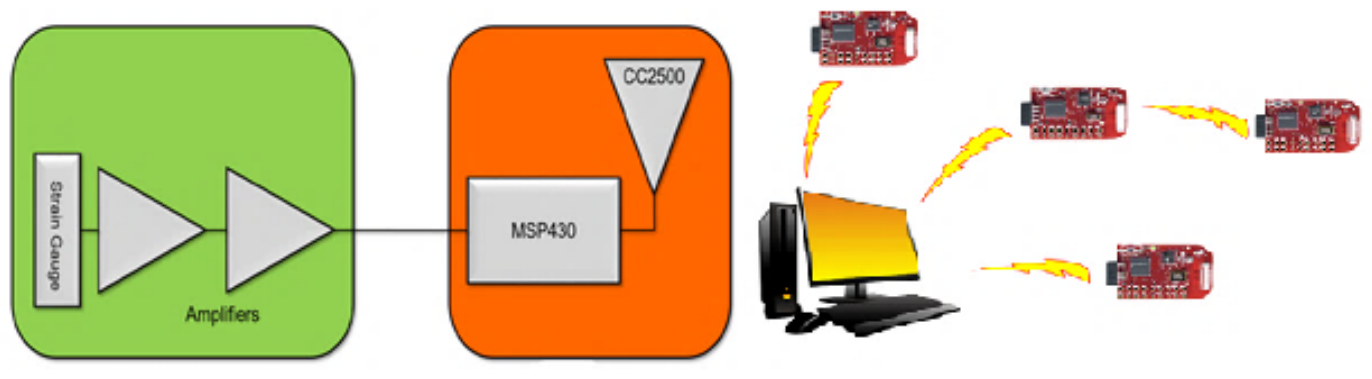

Figure 2.7 The schematic diagram of wireless signal transmission using MSPEZ430. 


\subsection{References}

1. Ekelof, Stig. "The genesis of the Wheatstone bridge". Engineering Science and Education Journal 10.1 (2001): 37-40.

2. David Harris \& Sarah Harris (2012). "Digital Design and Computer Architecture, Second Edition”, p. 515. Morgan Kaufmann. ISBN 0123944244.

3. Motchenbacher, Curtis D., and Joseph Alvin Connelly. "Low-noise electronic system design”. New York: Wiley, 1993.

4. AD8236, Analog Devices, Revision History: 5/09 - Revision 0: Initial Version.

5. Joffe, Elya B., and Kai-Sang Lock. "Grounds for grounding: a circuit to system handbook. John Wiley \& Sons, 2011.

6. Wilmshurst, Trevor H. Signal recovery from noise in electronic instrumentation. CRC Press, 1990.

7. MCP6441, Microchip Technology 2010-2012, ISBN: 978-1-62076-244-8.

8. Semiconductor, O. N. "Linear \& switching voltage regulator handbook". ON Semi (2002).

9. MCP 1700T, Microchip Technology, Revision History:10/2013.

10. TLV-711, Texas Instruments, SBVS142A -JULY 2010-REVISED AUGUST 2010.

11. Fette, Bruce A., et al. RF \& Wireless Technologies: Know It All. Elsevier, 2007.

12. EZ430-RF2274, Texas Instruments, Application Report, SLAA378D-December 2007-Revised April 2011. 


\section{Digital Potentiometer to Balance Wheatstone Bridge}

A digital potentiometer is a digitally controlled electronic component that mimics the functions of an analog potentiometer. They are built either from a resistor ladder integrated circuit or a digital-to-analog converter. Commonly used digital potentiometer is made by resistor ladder construction where each step (tap) in the ladder has their own switch which can connect this step to the output terminal of the potentiometer and the selected tap determines the resistance ratio of the potentiometer. The number of taps is indicated by bit values, an 8-bit potentiometer will have 256 taps (28) but potentiometers with resolution ranging from 5 to 10 bits are commercially available. Most of the potentiometer uses digital protocols like $\mathrm{I}^{2} \mathrm{C}$ or Serial Peripheral Interface Bus for communication [1]. The sensor system presented here incorporates an AD5110 digital potentiometer to eliminate resistor mismatches in the Wheatstone's Bridge and for offset adjustments.

\subsection{AD 5110}

AD 5110 is a single channel, $10 \mathrm{~K} \Omega$, non-volatile, 7-bit (128 taps) digital potentiometer with a low resistor tolerance of $\pm 8 \%$. The output voltage can be varied by changing the wiper position and the wiper position is determined by the contents of the RDAC register. The RDAC is a standard logic register which can be programmed with any wiper position setting using the $\mathrm{I}^{2} \mathrm{C}$ compatible digital interface. The contents of the RDAC register can be stored in the EEPROM memory such that the specific wiper position is always restored for subsequent power-up. The storing of EEPROM data takes approximately $18 \mathrm{~ms}$; during this time the device is locked and does not acknowledge any new command, thereby preventing any changes to the resistor setting [2]. 


\subsubsection{RDAC Register and EEPROM}

As already mentioned, the RDAC register directly controls the positon of the wiper in the digital potentiometer. Figure 3.1 shows the 16-bit input-shift register which consist of five unused bits (set to zero), followed by three command bits (C2 $\mathrm{C} 1 \mathrm{C} 0$ ) that directs the potentiometer to perform various operations and it also controls the internal EEPROM and eight RDAC data bits (DB7-DB0).

\begin{tabular}{|l|l|l|l|l|l|l|l|l|l|l|l|l|l|l|l|}
\hline 0 & 0 & 0 & 0 & 0 & C2 & C1 & C0 & D7 & D6 & D5 & D4 & D3 & D2 & D1 & D0 \\
\hline
\end{tabular}

Figure 3.1 The input shift register content

Unlimited changes of resistor setting can be made by changing the contents of the $\mathrm{RDAC}$ register. It is possible to read from and write to the RDAC register using the $\mathrm{I}^{2} \mathrm{C}$ interface by setting the control bits to specific values. Table 3.1 depicts the command operation truth table for the control bits in the input shift register.

Table 3.1 Command Operation Truth Table

\begin{tabular}{|c|c|c|c|c|}
\hline $\begin{array}{c}\text { Command } \\
\text { Number }\end{array}$ & C2 & C1 & C0 & Operation \\
\hline 0 & 0 & 0 & 0 & No operation \\
\hline 1 & 0 & 0 & 1 & Write contents of RDAC to EEPROM \\
\hline 2 & 0 & 1 & 0 & $\begin{array}{c}\text { Write contents of serial register data to } \\
\text { RDAC }\end{array}$ \\
\hline 3 & 0 & 1 & 1 & Software shutdown \\
\hline 4 & 1 & 0 & 0 & Software reset \\
\hline 5 & 1 & 0 & 1 & Read contents of RDAC register \\
\hline 6 & 1 & 1 & 0 & Read contents of EEPROM \\
\hline
\end{tabular}

The RDAC of AD5110 has two-stage segmentation architecture to achieve optimum performance where the internal resistor $(10 \mathrm{~K} \Omega)$ is partitioned into two. 
The terminals A and B are connected to the separate segments and the wiper switch is designed with the transmission gate CMOS technology, the gate voltage is derived from applied VDD. In addition, the resistance tolerance error is saved within the EEPROM which can be read back and used to calculate the end-to-end tolerance [2].

\subsection{2. $I^{2} \mathrm{C}$ Serial Interface}

$\mathrm{I}^{2} \mathrm{C}$ (Inter-Integrated Circuit) is a multi-master, multi-slave, single-ended, serial bus which is used to connect low-speed peripheral ICs to processors or microcontrollers for intra-board communication. Serial Data Line (SDA) and Serial Clock Line (SCL) are two bi-directional, open-drain lines used in the $\mathrm{I}^{2} \mathrm{C}$ protocol and pull-up resistors are needed for both SDA and SCL. The AD 5110 operates as a slave device under the control of a master device and the $\mathrm{I}^{2} \mathrm{C}$ compatible serial interface of the potentiometer can be connected to an $\mathrm{I}^{2} \mathrm{C}$ bus of the master device. The AD5110 support standard $(100 \mathrm{kHz})$ and fast $(400 \mathrm{kHz})$ data transfer modes. The $\mathrm{I}^{2} \mathrm{C}$ serial communication protocol is as follows:

1) Data transfer is initiated by the master by sending a start condition (a highto-low transition on the SDA while SCL is high) to the slave.

2) The master sends an address byte consisting of seven-bit slave address followed by an R/W (Read or write). The slave device acknowledges to the transmitted address by pulling SDA low during the ninth clock pulse as shown in Figure 3.2. The address of the AD5110 is specified in the data sheet as 0101111 .

3) If the $\mathrm{R} / \mathrm{W}$ bit is set high, the master reads from the slave device. However, if the bit is set low, the master writes to the slave device.

4) Remaining data is transmitted over the serial bus in sequence of nine clock pulses (eight-bit data followed by an acknowledge bit). The transition on SDA should always occur at the low period of the SCL and remain stable during the high period of SCL as shown in Figure 3.2.

5) When all the data bits have been read, or written, the master initiates a STOP 
condition. In write mode, the master pulls the SDA line high during the tenth clock pulse to establish the stop condition. However, in read mode the SDA line remains high (no acknowledge by master during the ninth clock pulse). The master brings the SDA line low before the tenth clock pulse, and high during the tenth clock pulse to establish a stop condition [2,3].

\subsubsection{AD5110 Pin Configuration}

The AD5110 is available in a $2 \mathrm{~mm} \times 2 \mathrm{~mm}$ LFCSP package. A power supply voltage ranging from $2.3 \mathrm{~V}$ to $5.5 \mathrm{~V}$ when applied to VDD can be used to power the device and this pin need to be decoupled with $0.1 \mu \mathrm{F}$ capacitor to ensure device stability and protection.

Figure 3.3a illustrates the functional block diagram of AD5110 and Figure 3.3b shows the pin-out diagram of device. The SDA pin is used in conjunction with the SCL line (From the master) to clock data into or out of the 16-bit input registers and SCL pin is used in conjunction with the SDA line to clock data into and out of the register. Both the SDA and SCL are bidirectional, open-drain data lines that should be pulled to the supply with external pull-up resistors. The Logic power supply (pin 8) can be used to reset the device as shown in Figure 3.3a need to be decoupled with a $0.1 \mu \mathrm{F}$ capacitor. To turn ON the device VLOGIC should be connected to a positive power supply as specified in Table 3.2 and any voltage below this range will turn OFF the device $[2,3]$. The wiper position had to be saved to the internal EEPROM to restore the wiper output once the device is switched back ON or else the master again must transmit the data to AD5110 to return the wiper to the desired position.

Table 3.2 Pinout Description

\begin{tabular}{|l|l|l|}
\hline Pin No & Mnemonics & Description \\
\hline 1 & $\mathrm{~V}_{\mathrm{DD}}$ & Positive power supply $(2.3 \mathrm{~V}-5.5 \mathrm{~V})$ \\
\hline 2 & $\mathrm{~A}$ & Terminal A of RDAC $\left(\mathrm{GND} \leq \mathrm{V}_{\mathrm{A}} \leq \mathrm{V}_{\mathrm{DD}}\right)$ \\
\hline
\end{tabular}




\begin{tabular}{|l|l|l|}
\hline 3 & W & Wiper Terminal of RDAC $(\mathrm{GND} \leq \mathrm{VW} \leq \mathrm{VDD})$ \\
\hline 4 & B & Terminal B of RDAC $\left(\mathrm{GND} \leq \mathrm{V}_{\mathrm{B}} \leq \mathrm{V}_{\mathrm{DD}}\right)$ \\
\hline 5 & GND & Ground pin, reference ground. \\
\hline 6 & SCL & Serial Clock Line \\
\hline 7 & SDA & Serial Data Line \\
\hline 8 & VOGIC & Logic Power supply $\left(1.8 \mathrm{~V}-\mathrm{V}_{\mathrm{DD}}\right)$ \\
\hline
\end{tabular}

\subsection{Programming the AD5110 Digital Potentiometer}

An ez430-rf2274 microcontroller is used as the master device to control the digital potentiometer. It is a USB-based MSP430 wireless development tool that includes an MSP430F2274 microcontroller and a $\mathrm{CC} 2500$ 2.4-GHz wireless transceiver. The MSP430 handles serial communication using an on-chip peripheral called USCI (Universal Serial Communication Interface). The peripheral handles multiple synchronous and asynchronous serial communication formats using USCI_A0 and USCI_B0 modules. The USCI_B0 module supports the $\mathrm{I}^{2} \mathrm{C}$ mode of communication. Any components connected to the $\mathrm{I}^{2} \mathrm{C}$ bus serially transmit or receive data from the USCI_B0 module through the two-wire $\mathrm{I}^{2} \mathrm{C}$ interface, these components are recognized by a unique address and they can operate as either a transmitter or a receiver. The master initiates the data transfer and generates the clock signal SCL for other components connected to the $\mathrm{I}^{2} \mathrm{C}$ bus and any device addressed by the master is considered as slave. $\mathrm{I}^{2} \mathrm{C}$ data is communicated through SDA and SCL pins which are connected to the positive power supply using pull-up resistors [4].

Programming the digital potentiometer starts with initializing the USCI module of the MSP430 by setting the UCSWRST bit of UCBxCTL1 control register. The important control registers and there bit descriptions are given in the Table. After the initialization, the USCI module is released for operation by clearing the UCSWRST bit. Then the master transmitter mode is initiated by writing the slave address to the UCBxI2CSA register, setting UCTR for transmitter mode and setting UCTXSTT to 
generate a start condition. The USCI checks for bus availability and, generates the bus availability and transmits the slave address. The transmit interrupt is enabled by setting the UCTXIFG bit and after generating the start condition the first data set can be stored into the transmit buffer (UCBxTXBUF) of the USCI module. The data written into UCBXTXBUF is transmitted if mediation is not lost during transmission of the slave address. The UCTXSTT bit is cleared as soon as the master receives the acknowledgement from the slave. Similarly, the rest of the bytes will be transmitted serially to the slave device as shown in the Flowchart 3.1. After transmitting every data to the slave, a stop condition is generated after the acknowledgement from the slave by setting the UCTXSTT $[5,6]$.

Table 3.3 Control registers of USCI module, their descriptions and user assigned values for initializing the USCI module of the MSPEZ-430

\begin{tabular}{|c|c|c|c|}
\hline \multicolumn{5}{|c|}{ UCB0CTL0 } & VALUE \\
\hline BIT & FIELD & DESCRIPTION & $1 \mathrm{~b}$ \\
\hline 3 & UCMST & Master/ Slave mode select & $11 \mathrm{~b}$ \\
\hline $2-1$ & UCMODEx & USCI mode select & $1 \mathrm{~b}$ \\
\hline 0 & UCSYNC & Synchronous mode enable & VALUE \\
\hline \multicolumn{5}{|c|}{} & UCB0CTL1 & $10 \mathrm{~b}$ \\
\hline BIT & FIELD & DESCRIPTION & $1 \mathrm{~b}$ \\
\hline $7-6$ & UCSSELX & USCI Clock select & VALUE \\
\hline 0 & UCSWRST & Software reset enable & 12 \\
\hline & & UCB0BRO & VALUE \\
\hline BIT & FIELD & DESCRIPTION & 0 \\
\hline $7-0$ & UCBR0 & Bit Clock Pre-scaler low byte & UCB0BRI \\
\hline BIT & FIELD & DESCRIPTION & \\
\hline $7-0$ & UCBR1 & Bit Clock Pre-scaler high byte & \\
\hline
\end{tabular}


A customizable program is compiled and downloaded into the digital potentiometer using IAR Embedded Workbench. The dedicated SDA (pin 18) and SCL (pin 15) pins of the master device is connected to the corresponding serial communication lines of AD5110. The data flow between the master and the slave is depicted in the Flow chart 1 . The master initiates the communication by establishing a start condition (bringing the data line low while SCL is high). The master then sends a single address byte (5E) stored in its dedicated $\mathrm{I}^{2} \mathrm{C}$ address register to the slave in eight clock pulses. The address byte consists of seven-bit slave address and a read or write bit at the end, the read or write bit is set low so that the master can write the data into the slave. If the seven-bits matches the slave address the slave acknowledges the transfer by pulling SDA low during the ninth clock pulse and it waits for the next set of data bytes. The master serially sends two bytes of data packet to the slave; the 16-bit word consists of five unused bits (zero bits) followed by three control bits and eight RDAC bits. The contents of the serial data are loaded to the $\mathrm{RDAC}$ register by setting the control bit as $0 \mathrm{x} 02$. 


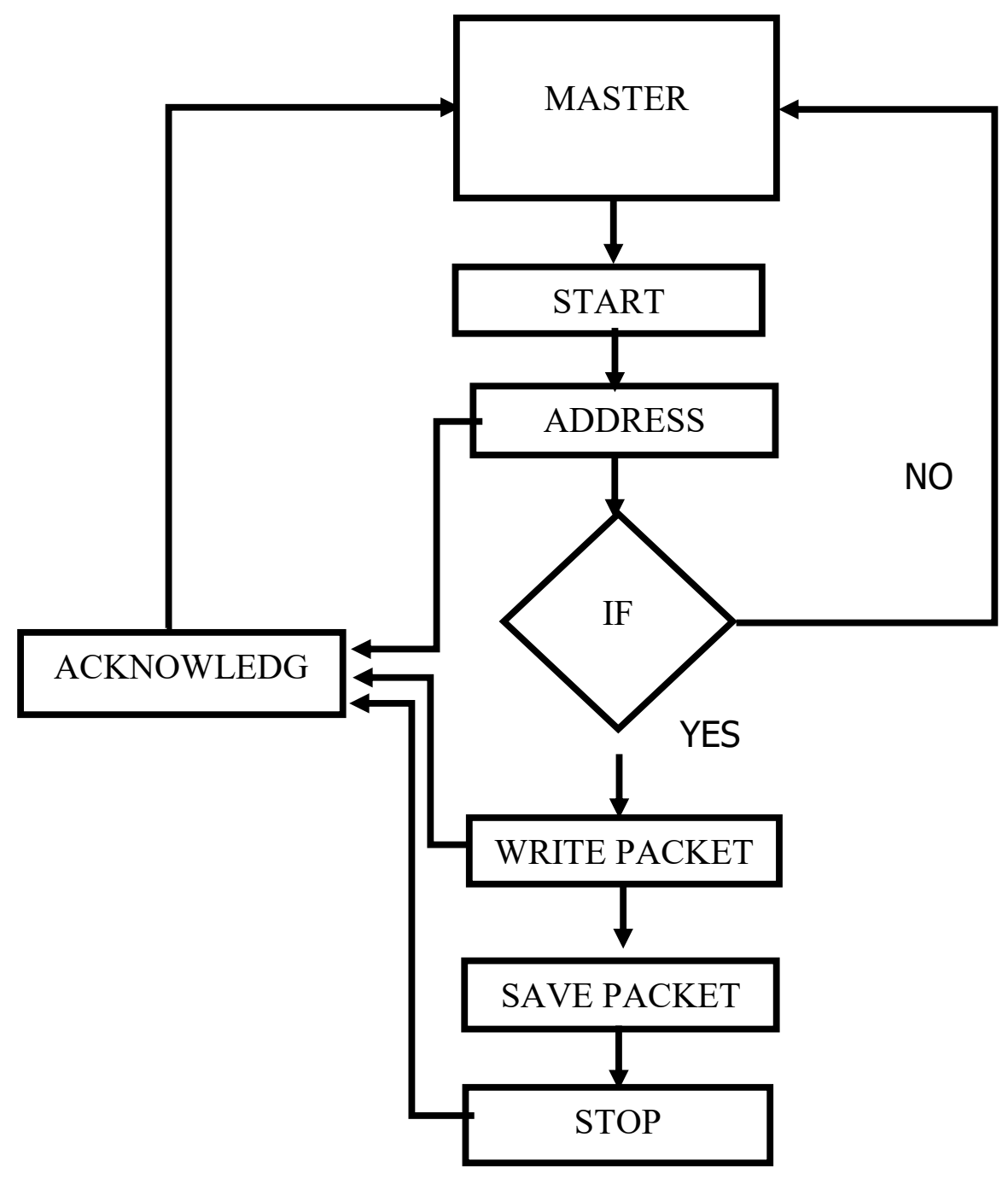

Flow chart 3.1 Data-flow in AD5110 
The eight-bit data bits determine the position of the wiper. For example, if the RDAC register is loaded with $0 \times 3 \mathrm{~F}$ (128-taps), the wiper is connected to full-scale of the variable resistor and there is no limit in changing the RDAC contents. After setting the wiper into a desirable position the contents of the RDAC can be stored into the internal EEPROM memory so that the wiper position can be reestablished for subsequent power-ups. The contents of the RDAC is finally saved into the EEPROM by loading the control bits with specified binary value (001). The master establishes a STOP condition at the end of the data transfer $[4,5,6]$.

\subsection{Application of AD5110 Digital Potentiometer}

Analog mechanical potentiometers are prone to performance changes and reliability concerns over time. The mechanical wiper contact resistance can change due to oxidation, aging and wear reducing the usable life-time of the mechanical potentiometers. They are highly sensitive to shock and vibrations. A digital potentiometer consists of a series of CMOS transmission gates and due to the absence of mechanical components the digital potentiometer offers better reliability and stability. They are also resilient against shocks, vibrations, aging and contact as compared to analog mechanical potentiometers. However, some of the advantages of mechanical potentiometer over their digital counter-parts include the ability to withstand high voltages, larger current carrying capability and larger power generation [7].

The sensor system presented here has low power requirement and they are intended to perform reliably over a longer time frame. The analog potentiometer is replaced by an AD5110 digital potentiometer to ensure better stability and reliability. The SDA and SCL pins are connected to the corresponding $\mathrm{I}^{2} \mathrm{C}$ pins of the microcontroller (MSPEZ430-RF2274) and the component is powered by applying a $2.5 \mathrm{~V} \mathrm{DC}$ voltage from the voltage regulator. The maximum resistance offered by the analog potentiometer is $2 \mathrm{~K} \Omega$ and that offered by the AD5110 is $10 \mathrm{~K} \Omega$. Replacing 
the analog potentiometer with the digital causes huge changes in the resistance ratio of the Wheatstone bridge. So, appropriate resistor values need to be selected for the Wheatstone bridge to accommodate the changes caused by replacement. The schematic diagram shown in figure depicts the updated Wheatstone bridge configuration with the digital pot.

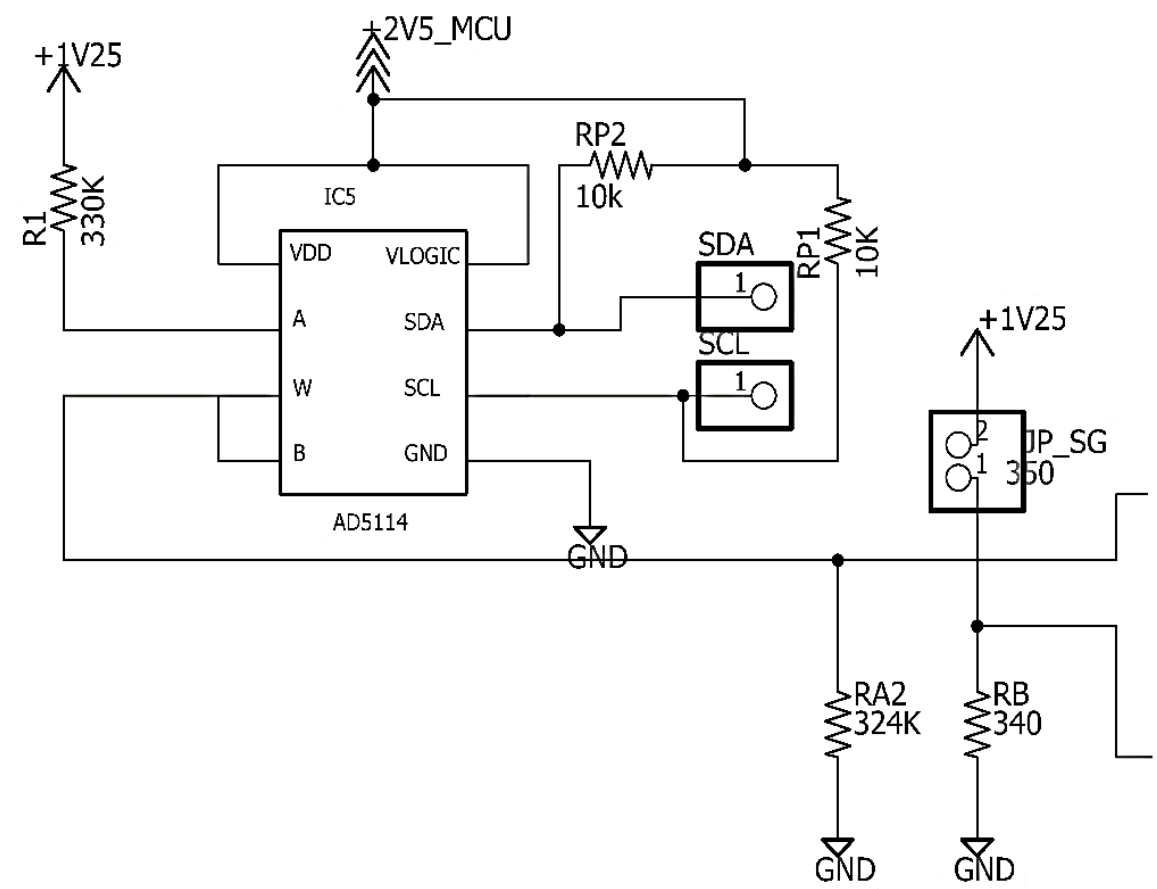

Figure 3.2 The circuit diagram of the Wheatstone bridge with AD5110 connected to a $330 \mathrm{~K} \Omega$ resistor and $324 \mathrm{~K} \Omega$ resistor (instead of two $56 \mathrm{~K} \Omega$ ). The voltage dropping across the digital pot and the upper $330 \mathrm{~K} \Omega$ resistor is fed to the noninverting input of the instrumentation amplifier and the voltage drop across the strain gauge is applied to the inverting input.

Initially, an appropriate wiper position is selected such that the sensor system has an offset voltage at its output pin. The position of the wiper is stored in the internal EEPROM memory of AD5110 digital potentiometer such that the wiper position is restored for subsequent power up. The master device is then reprogrammed with the 
end device code so that it transmits the strain data to the access point connected to the PC where a customizable Matlab GUI displays the collected data. 


\subsection{References}

1. http://www.analog.com/en/technical-documentation/Resources/digital-toanalog-converters/digital-potentiometers/listing.html.

2. http://www.analog.com/media/en/technical-documentation/datasheets/AD5110_5112_5114.pdf.

3. Patel, J. J., and Prof BH Soni. "Design and Implementation of I2c Bus Controller Using Verilog." Proc. Journal of Information, Knowledge and Research in Electronics and Communication Engineering ISSN: 0975-6779.

4. Instruments, Texas. "eZ430-RF2500 development tool user's guide". Texas Instruments SLAU227E (2009).

5. Litovsky, Gustavo. "Beginning Microcontrollers with the MSP430 Tutorial", Texas Instruments (2010).

6. Morales, Miguel, and Zachery Shivers. "Wireless sensor monitor using the eZ430-RF2500."Texas Instruments (2007): 15.

7. http://powerelectronics.com/electromechanical/digital-vs-mechanicalpotentiometers-design-considerations-maximize-system-perform. 


\section{Sensor Performance}

\subsection{Mechanical Testing}

This section describes the response of the strain gauge sensor when different forces were applied to the bone fixation plate. Test Resources 100 series, an electromechanical loading test system equipped with $1000 \mathrm{lbs}$. load cell was used to perform compression testing on the bone fixation plate. The bone fixation plate was secured vertically in an enclosure on the mechanical loader before starting the compression testing. This ensured that the load was applied normal to the top surface of the plate. The loader was configured for different tests by specifying the end force and loading rate. Figure 4.1 plots the measured sensor voltage when force was applied from 0 to $250 \mathrm{~N}$ at a rate of $1000 \mathrm{~N} / \mathrm{min}$, and back to $0 \mathrm{~N}$ at the same rate. The baseline voltage of the sensor was set as $0.573 \mathrm{~V}$ by setting the digital potentiometer's resistance. The sensor output reached a maximum value of 0.8569 $\mathrm{V}$ at the peak load of $250 \mathrm{~N}$ and returned to its initial base voltage following the load from the mechanical loader. The plot shows the sensor system successfully tracked the change in load on the external fixation plate when applying and removing load.

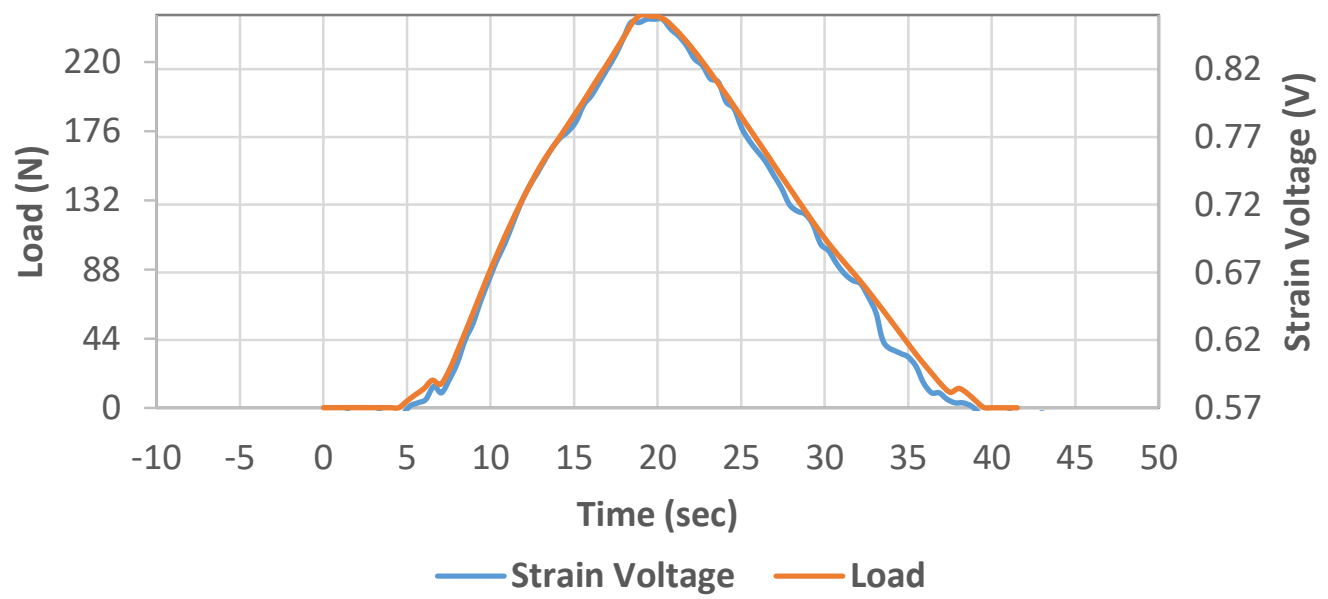

Figure 4. 1 The sensor response to both increasing and decreasing loads

Figure 4.2 depicts the sensor response against a varying load over three trials. The 
plot shows consistent response of the sensor for same loading conditions. However, a minor drift in the base-voltage was observed before the start of each trial (as shown in Figure 4.2) this shift was also observed in the peak value for all the three trials.

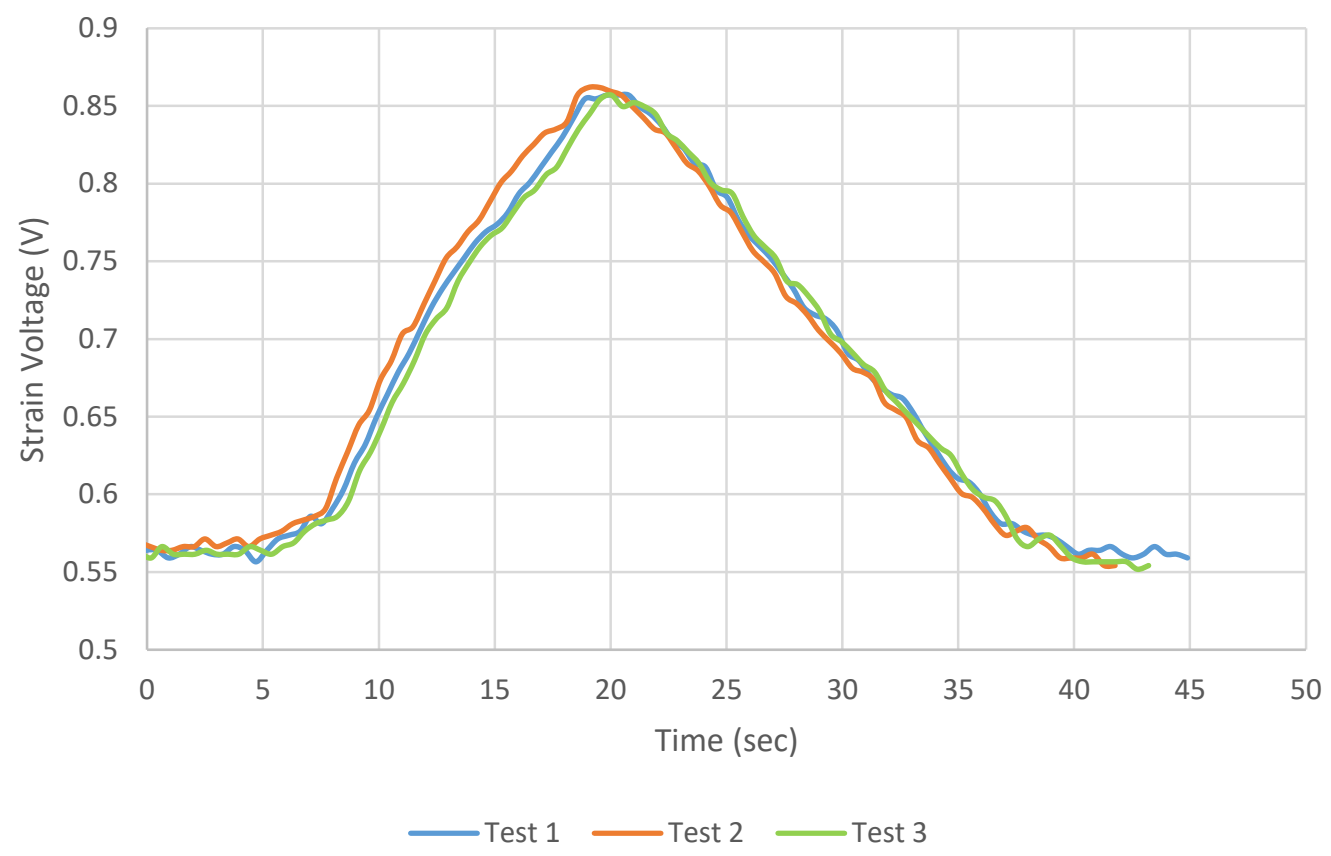

Figure 4. 2 The sensor response to both increasing and decreasing loads for multiple trials

\subsection{Sensor Response for Compressive Loads}

Figure 3.3 shows that the strain output voltage varied directly proportional to with the applied force on the external fixator plate. The sensitivity of the sensor was obtained as $1 \mathrm{mV} / \mathrm{N}$ using Equation (1).

$$
\text { Sensitivity }=\frac{\Delta V_{\text {OUT }}}{L}
$$

Where, $\Delta V_{\text {OUT }}$ is the change in output voltage of the sensor when the bone plate was loaded from 0 to $250 \mathrm{~N}$. 
$L$ is the total amount of load applied to the bone plate.

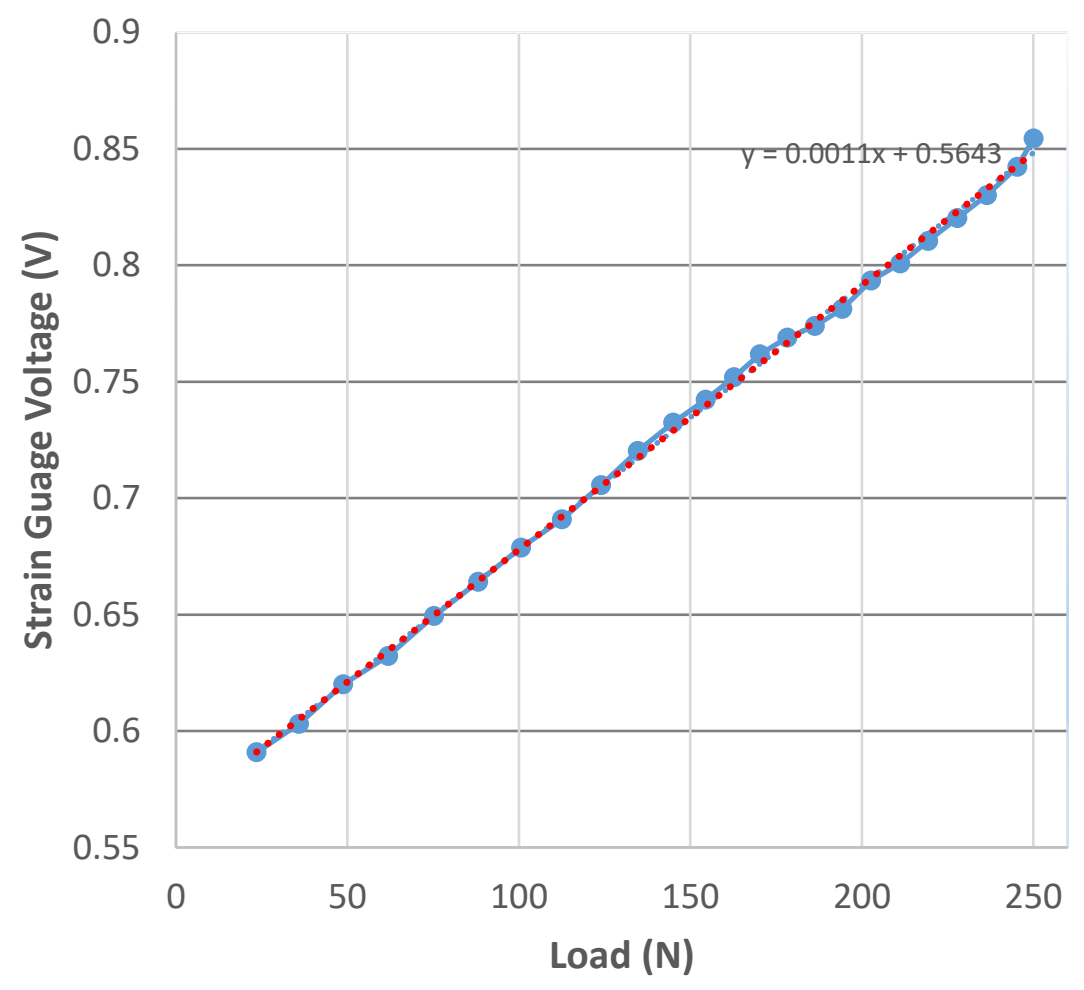

Figure 4. 3 The graph showing the linear relationship between the sensor voltage and the applied load. From the trend line the linearity is calculated as $96.05 \%$.

The applied force was calibrated from the strain voltage by obtaining the coefficient of the first order polynomial as shown below:

$$
F=m * V+c
$$

where $F$ is the applied force in Newton and $V$ is the corresponding output voltage from the sensor and Figure $\mathbf{4 . 3}$ depicts the relationship between these two variables. Linear regression was used to calculate the value of the variables the average slope $m$ and $y$-intercept $c$, as 830.45 and -463 respectively and the following equation was obtained by plugging these values into the Equation (1). 


$$
F=830.45 * V-463
$$

Equation (3)

The strain gauge voltage was calibrated to force using Equation (3) and Figure 3.3 depicts the relationship between the estimated load with the actual applied load.

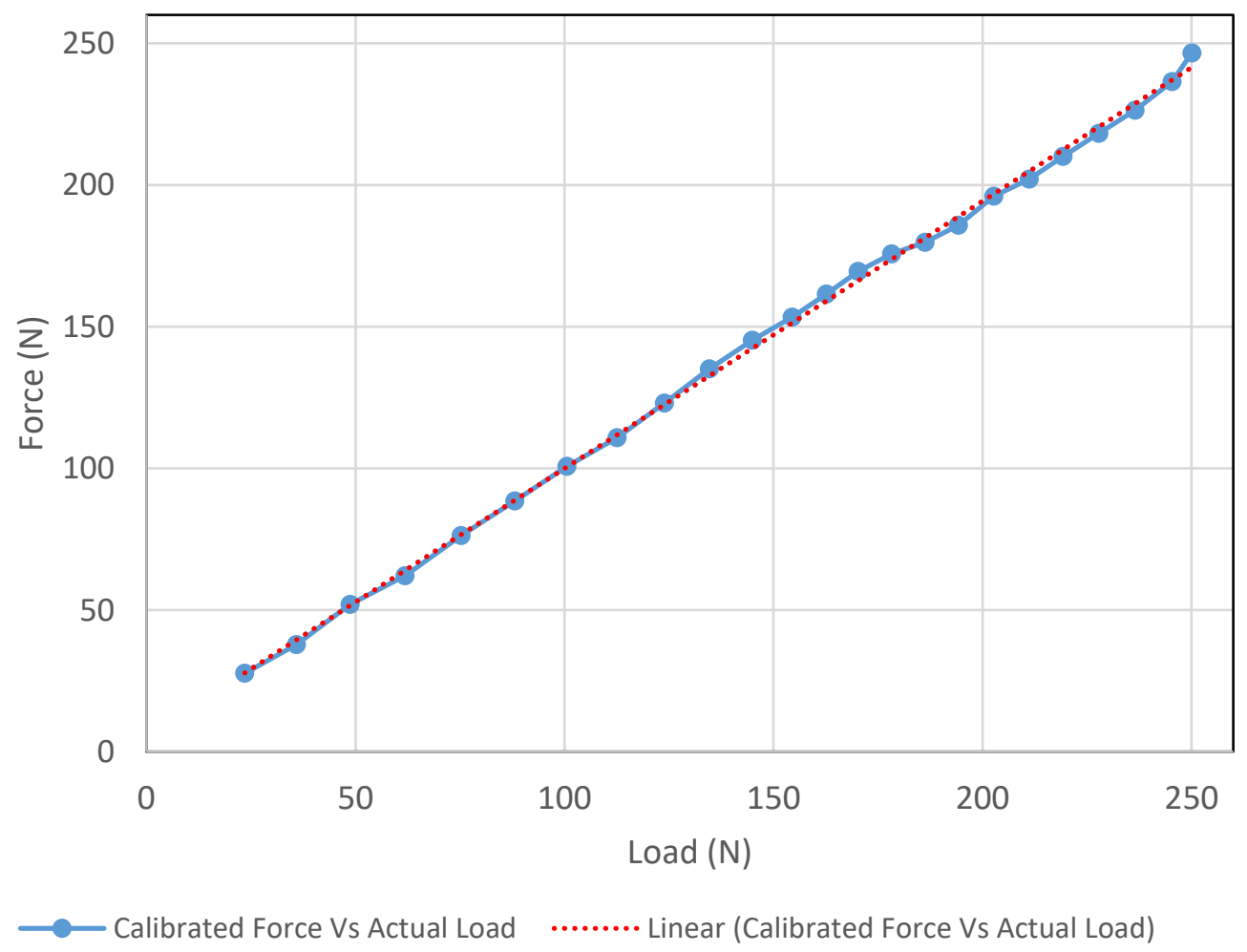

Figure 4.4. The load was calculated from the strain voltage data by linear regression and plotted against the load applied by the mechanical loader.

The voltage resolution of the sensor system was obtained using Equation (4).

$$
\text { Voltage resolution }=\frac{V_{I}}{2^{N}}
$$

Where $V_{\text {I }}$ is the input voltage to the microcontroller.

$N$ is the number of ADC converter bits available in the microcontroller. 
The microcontroller used for data collection equipped with 10-bit analog-todigital converter with a voltage scale of $0-2.5 \mathrm{~V}$ (quantized to $2^{10}$ or 1024 levels). Using these values in Equation (3) the voltage resolution of the system was calculated as $2 \mathrm{mV}$. The force resolution of the sensor was obtained as $2.27 \mathrm{~N}$ using the following equation:

$$
\text { Force resolution }=\frac{\text { Voltage resolution }}{\text { Sensitivity }}
$$

\subsection{Mechanical Testing Under Cyclic Loading}

The repeatability exhibited by the sensor system was demonstrated by applying several cycles of loading to the external fixation system. Figure 4.5 depicts the results of cyclic loading the external fixator between $0 \mathrm{~N}$ to $250 \mathrm{~N}$ over 10 cycles at $1000 \mathrm{~N} / \mathrm{min}$. As shown in the figure the sensor system exhibited good repeatability and stability with minimal drift after the cyclic loading.

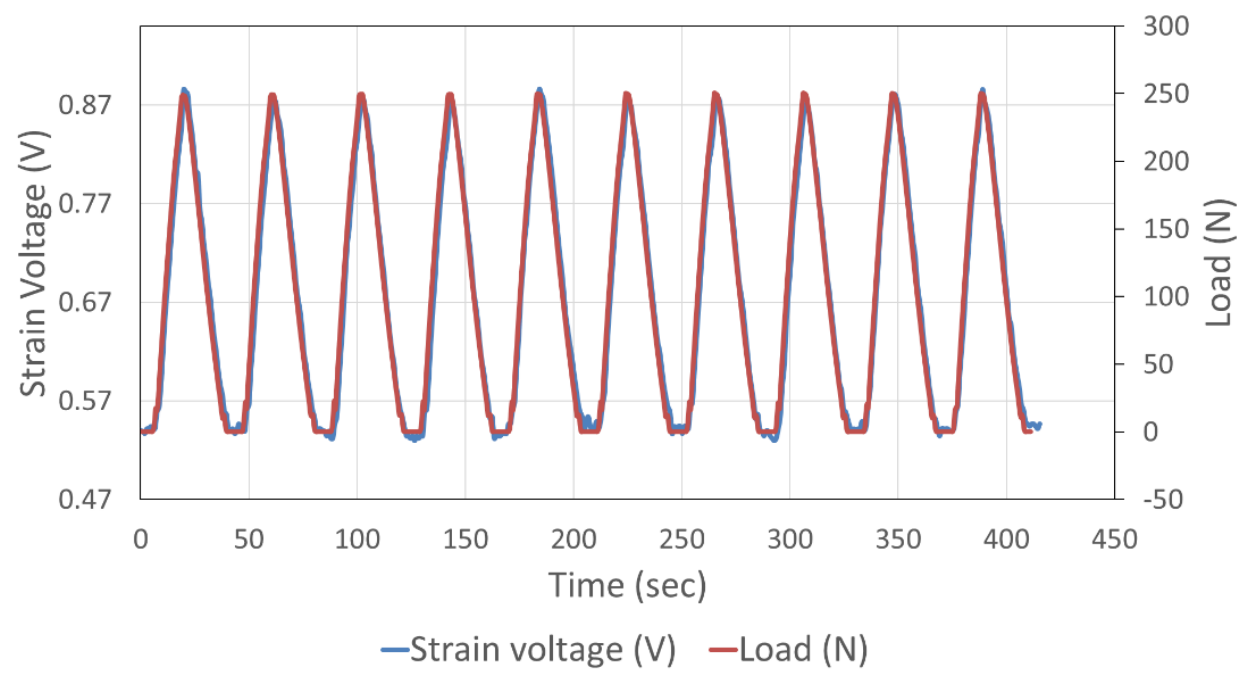

Figure 4.5: Repeatability and stability exhibited by sensor system for 10 cycles of loading between 0 to $250 \mathrm{~N}$.

The sensor tracked the loading profiles from the mechanical loader at the highest peak value. The overall change in the strain voltage from the baseline value to the 
peak load was calculated as $0.3418 \mathrm{~V}$.

\subsection{Baseline Shift}

It was observed that each sensor system did not go back to the initial base voltage after compressive loading. Further testing showed that each newly adhered strain sensor on the bone plate surface needed an initial pre-straining to stop the baseline voltage from shifting The bone fixation plate equipped with the sensor system was subjected to cyclic compressive load between 0 to $250 \mathrm{~N}$ at $1000 \mathrm{~N} / \mathrm{min}$. The difference between the baseline voltage and the final voltage was recorded to calculate baseline shift. This procedure is repeated for 10 cycles with a sixty-second delay in between each cycle.

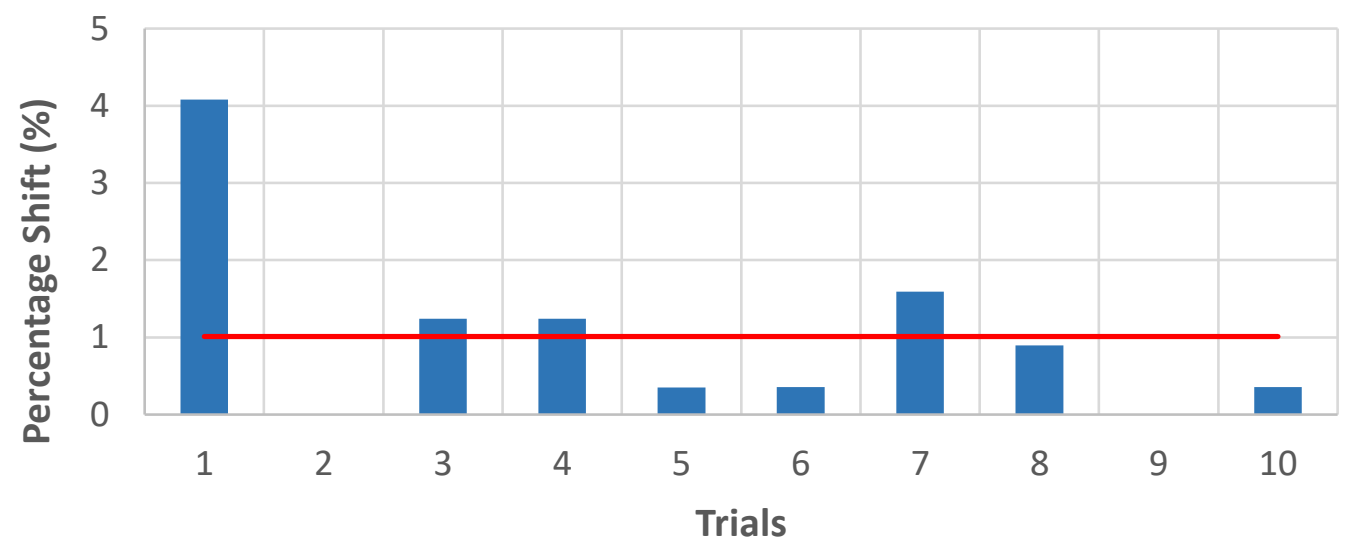

Percentage Shift —-Average Shift

Figure 4. 4 The percentage shift in base voltage for each trial lies within a range of $0-4 \%$. The average error is calculated as $1.01 \%$

Figure 4.6 depicts the percentage shift in the baseline voltage after subsequent cyclic loading. It was noted that considerable shift occurred only during the first trial around $4 \%$. However, for subsequent trials the shift is found to be less than half of the initial trial. This observation corroborates the need of a pre-straining to reduce 
the baseline-shift. It was also noted that the magnitude of noise interference in the signal was needed to assess the sensor performance. And to measure the noise level, the sensor system was turned on with no load applied, as depicted in Figure 4.7. The measured maximum deviation of the base voltage due to noise was $\pm 10 \mathrm{mV}$.

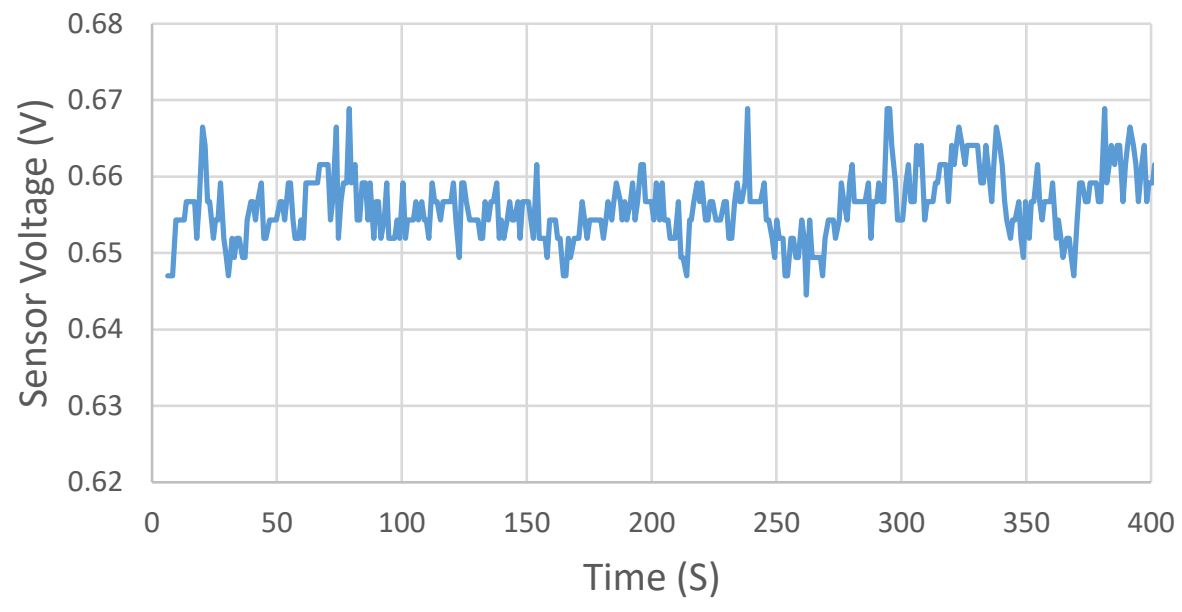

Figure 4.7: The presence of noise in the system under no load condition. The deviation of the base voltage due to noise is estimated as $\pm 10 \mathrm{mV}$.

\subsection{Temperature Testing}

The effect of temperature on the strain gauge was also experimentally assessed. Figure 4.7 shows the deviation in the baseline voltage under the no-load condition with varying temperature $\left(19{ }^{\circ} \mathrm{C}-23{ }^{\circ} \mathrm{C}\right)$ for 14 hours. The thermistor voltage corresponds to the change in temperature during the period of the experiment. The test revealed that the sensor voltage is dependent on temperature swings surrounding it. 


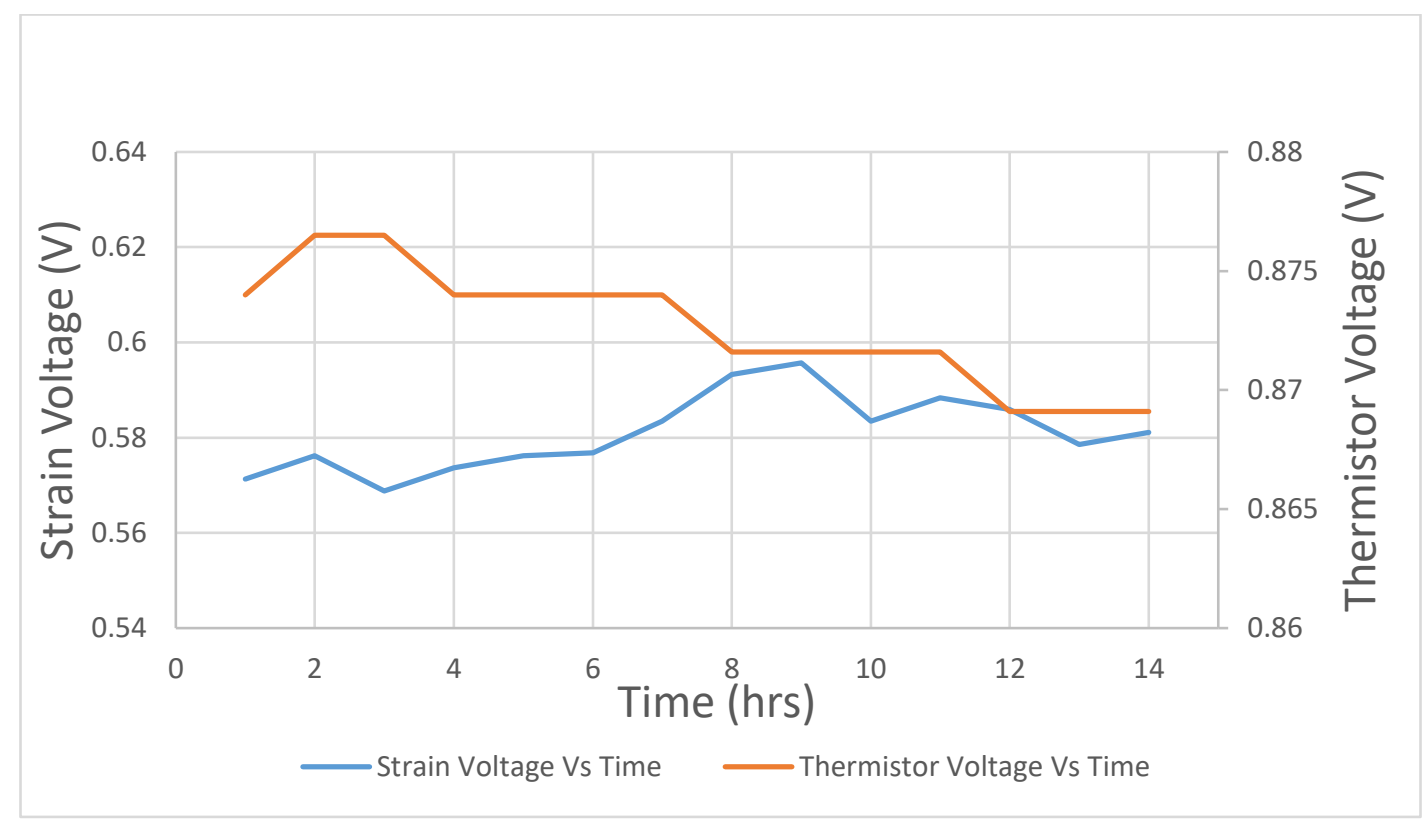

Figure 4. 8 The variation in temperature and strain gauge voltage.

The temperature dependency of the strain measurement was further examined by placing the sensor in temperature controlled environments. The sensor output voltage was monitored at different temperature conditions and the bone plate was not stressed during the test so that the shift in output voltage only attributed to thermally induced strain. The sensor response was recorded when the system was placed in an incubator, a freezer and at room temperature. Thus, the bone plate was subjected to temperature swings ranging from $-6^{\circ} \mathrm{C}$ to $37^{\circ} \mathrm{C}$. The strain data, temperature data and actual temperature of the bone plate (measured using a thermometer) were noted at each step.

Initially, the external fixator plate equipped with the sensor system was kept in an incubator for 30 minutes. In the second stage of the experiment, the external fixator plate was kept at room temperature for 30 minutes. The setup was then transferred to a freezer where the steady drop in temperature resulted in a similarly steady increase in output voltage as depicted in Figure 4.8. The change in the thermistor output voltage per unit change in temperature was curve-fit with a linear equation and determined as $15 \mathrm{mV} /{ }^{\circ} \mathrm{C}$. Similarly, the strain gauge voltage due to 
temperature variations was measured and curve-fit as $15.6 \mathrm{mV} /{ }^{\circ} \mathrm{C}$. For application, such as monitoring strain at an external bone fixation plate, the strain gauge voltage variation due to temperature should be calibrated.

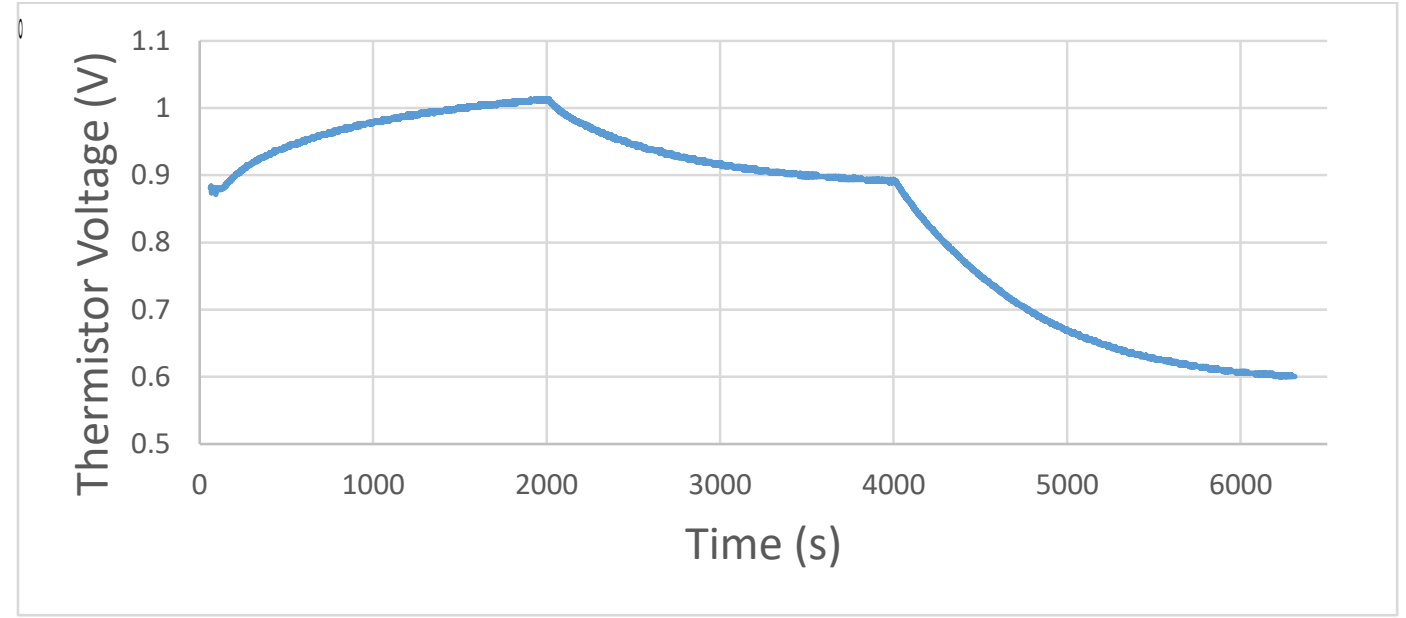

Figure 4.8 The change in thermistor voltage when the system was kept at different temperature settings. 


\section{Discussions}

The sensor system demonstrates its ability to track the changes in load when the external fixator plate is subject to compressive force between 0 and $250 \mathrm{~N}$ at 1000 $\mathrm{N} / \mathrm{min}$. The end force of $250 \mathrm{~N}$ was selected because it is considered as the maximum amount of load an external fixator plate can endure when used in an ovine model. However, the sampling rate of the mechanical loader and the sensor system are different and it was difficult to trigger the both systems at the exact same time. To plot both the load and the strain voltage on the same axis the time was normalized, minor deviation that can be seen in the plots are due to normalization.

The overall change in the sensor voltage during loading was found to be 0.2832 $\mathrm{V}$. The peak voltage was found to be only $43 \%$ of the maximum achievable output which means that the range offered by the sensor system is appropriate for the intended application and they can even be employed for strain measurements beyond the normal conditions $(0-250 \mathrm{~N})$ without causing output voltage saturation. Slight deviations were observed between each trial even when the loading profile of the loader was kept unchanged. This slight deviation can be attributed to the corresponding shift in base voltage which was evident before each trial. It was observed that the mechanical loader will recalibrate the position itself before each trial and this protocol will induce a finite amount of pre-stress to the external fixator plate which might be a reason for the observed shift in base voltage. The shift can also be attributed due to the noise in the environment, and the continuous loading of the external plate can cause mechanical hysteresis.

The linearity of the system was assessed by plotting the actual load with the sensor output voltage. The sensor exhibited good linear relationship with $96.05 \%$ linearity. The sensor output voltage was calibrated to the load using linear regression. Figure 4.4 shows this relationship between the actual load (from the mechanical loader) and the calibrated load. The trend line indicates that the deviation of the sensor output to the corresponding applied load is minimal. The force resolution of 
the sensor system was calculated as $2.21 \mathrm{~N}$ which is adequate for the intended application.

The system also exhibited good repeatability and stability when subjected to 10 compressive loading cycles as no significant drift was observed from the plot (Figure 4.5). During the first test, the sensor output exhibited shift from the base voltage. However, pre-straining the bone plate considerably reduced the shift in baseline voltage. The observed trend can be explained by considering the anelastic effect of the strain gauge. Experimental studies have confirmed that the amount of recoverable strain depends highly on the pre-strain [2]. The error percentage in baseline shift observed for our experiment is around 4\% during the pre-strain but for every other test the post-test the error percentage dropped below $2 \%$. The average error was found to be $1.01 \%$ however, neglecting the error from the pre-stress will reduces the average error to $0.63 \%$. Thus, the shift in base voltage can be reduced significantly by exposing the sensor system to proper pre-straining before an actual test. The noise interference was a major cause of worry for the system and the experimental results depict the noise can induce a deviation of $\pm 11 \mathrm{mV}$ to the sensor response. With a sensitivity of $1.1 \mathrm{mV} / \mathrm{N}$, the noise signal alone can contribute to about $20 \mathrm{~N}$ error for the observed sensor response. However, no significant errors where observed during the load test which implies that the sensor system presented here has the capability to produce reliable results with an accuracy of $\pm 11 \mathrm{mV}$. It was observed that introducing a $100 \mathrm{nF}$ capacitor across the input and the ground terminal of the digital potentiometer reduced the noise level significantly. Future design must focus on ways to reduce the noise to minimum so that the accuracy of the system can be greatly enhanced. Another method to reduce noise is to perform a faster sampling in the microcontroller and averaging the output to the computer.

The sensors stability over time was evaluated by constantly running the system for 14 hours under no-load condition and variation in the sensor output observed can be attributed to the fluctuation in room temperature. Ideally the resistance of the strain gauge must only respond to the applied strain. However, strain 
gauge material, the lead wiring, all responds to changes in temperature which can bring significant amount of error in the strain measurement [3]. In our work the effect of thermally induced strain on the strain gauge was measured experimentally by monitoring the sensor output voltage with varying temperatures. The average change in sensor response with unit change in temperature was measured as $15.3 \mathrm{mV} /{ }^{\circ} \mathrm{C}$. While the temperature dependency of the strain gauge measurements does not contribute problems for internal bone fixation plate, for an external bone fixation plate, the strain gauge voltage variation due to temperature will need to be calibrated from the measure temperature value.

One of the major contribution of this project is to replace the analog potentiometer with a digital potentiometer. During a typical implantation procedure, the bone plate is screwed to the broken bone to stabilize the fracture which induces significant amount of pre-stress on the implant. The magnitude of pre-stress can induce huge drift in the sensor baseline, it can even saturate the sensor. As the magnitude of this surgery induced pre-stressing is impossible to quantify before the implantation, the base voltage calibration should be carried out after the implantation. Although the base voltage can be adjusted using the analog potentiometer it is impractical after the surgery. Hence, a digital potentiometer is used where the resistance is software controllable. But the sensor system with the digital potentiometer is found to be more susceptible to temperature fluctuation. Hence, it is better suited for internal plates. Temperature calibration routine is needed to remove the temperature dependency when used on an external plate. 


\section{Conclusion}

Real-time monitoring of implant sites can significantly enhance treatment efficacy. The results from experiments proved that the strain gauge based sensor system is capable of continuously monitoring real time stress and load acting on an external fixation plate. An external fixator plate equipped with the sensor system is subjected to compressive forces. The results indicate that the sensor system successfully tracked the changes in load on the external plate.

Fracture healing is a complex physiological process which usually results in the formation of a new bone that is anatomically and mechanically similar to prefractured bone. However, the fracture healing process can fail due to several reasons leading to non-union. Statistically, $10 \%$ of all fractures leads to non-union and this number goes up to $50 \%$ in long segmental defects (open-fractures) [4]. Current gold standard uses autologous grafts to enhance or induce bone healing in delayed cases. Although the success rate for these treatments is about $60-80 \%$, this treatment option may not be viable for all patient population due to various co-morbidity [5]. Growth factor treatment (rhBMP-2) had shown to induce bone formation in tibial non-unions at the same rate as observed with autologous grafts [6]. But, due to required supraphysiological doses, it is an unfeasible clinical option so far. During the dosage investigation studies in small rodents, it has been found out that the dosage can be significantly reduced by modulating the mechanical environment around the fracture [6]. Proper loading of segmental defects combined with growth factor treatment can significantly enhance the treatment efficacy. The sensor system presented in this thesis can be used to monitor the mechanical environment of the fracture. The platform's small, robust, and low power nature increases its usefulness in continuous wireless monitoring applications and actuation in many other applications. As a research tool, this device could be incorporated in studies to monitor the effect of Osseo-inductive biomaterials on bone healing to formulate new treatments in bone fracture healing. Clinically, they can be used as a complimentary tool to assess bone fracture along with radiographs or MRI. However, more research need to be carried 
out to enhance the reliability of the system. Furthermore, temperature dependence of the sensor system is the major cause of worry, thus future studies should focus on ways to reduce the temperature dependence of the system. 


\section{References}

1. Krinke, George J. The laboratory rat. Academic press, 2000.

2. Vuoristo, T., V-T. Kuokkala, and P. Peura. "Pre-strain dependent apparent Young's modulus of automotive sheets steels". Proceedings of the 2004 SEM X International Congress and Exposion on Experimental and Applied Mechanics, June 7-10, 2004 Costa Mesa, California USA. 2004.

3. "How Is Temperature Affecting Your Strain Measurement Accuracy?" National Instruments, Publish Date: Mar 15, 2016

4. Zimmermann, Gerald, and Arash Moghaddam. "Trauma: non-union: new trends." European instructional lectures”. Springer Berlin Heidelberg, 2010. 15-19.

5. Weise, K., and E. Winter. "Role of intramedullary nailing in pseudarthrosis and malalignment". Der Orthopad 25.3 (1996): 247-258.

6. Einhorn, Thomas A., and Stephen B. Trippel. "Growth factor treatment of fractures". Instructional course lectures 46 (1997): 483-486. 